\title{
Risk processes with shot noise Cox claim number process and reserve dependent premium rate*
}

\author{
Claudio Macci ${ }^{\dagger} \quad$ Giovanni Luca Torrisi ${ }^{\ddagger}$
}

\begin{abstract}
We consider a suitable scaling, called slow Markov walk limit, for a risk process with shot noise Cox claim number process and reserve dependent premium rate. We provide large deviation estimates for the ruin probability. Furthermore, we find an asymptotically efficient law for the simulation of the ruin probability using importance sampling. Finally, we present asymptotic bounds for ruin probabilities in the Bayesian setting.
\end{abstract}

AMS Subject Classification. 60F10, 91B30.

Keywords. Shot noise Cox process, large deviations, ruin probability, importance sampling, Bayesian Statistics.

\section{Introduction}

In risk theory literature, shot noise modelling usually occurs when dealing with the delay in claim settlement; see, for instance, Klüppelberg and Mikosch [KM1] and [KM2], Brémaud [Br] and the survey paper by Kühn $[\mathrm{K}]$.

Recently, Dassios and Jang [DJ1] and [DJ2] considered risk processes with constant premium rate and claim number process given by the superposition of a homogeneous Poisson process and a Cox process with a Poisson shot noise stochastic intensity (see Cox $[\mathrm{C}]$ and Cox and Isham [CI] for an introduction on Cox processes; see Møller [Mo] for applications of shot noise Cox processes in spatial statistics). An interpretation of this model is the following: in addition to "standard" claims, which occur according to a homogeneous Poisson process, there are also "induced" claims triggered by external events which lead to a sudden increase of the total claim. In particular, Dassios and Jang [DJ1] considered Cox processes driven by a Poisson shot noise stochastic intensity having a multiplicative form and an exponential shot shape. They used the theory of piecewise deterministic Markov processes to obtain the distribution of the aggregate claim amount under an equivalent Esscher measure. An estimation procedure for the stochastic intensity of the claim number process is considered in Dassios and Jang [DJ2].

The case of Cox processes driven by a general (i.e. not necessarily of multiplicative form and not necessarily with an exponential shot shape) Poisson shot noise stochastic intensity is considered in Albrecher and Asmussen [AA], where the infinite and finite horizon ruin problem is analyzed both for light-tailed and heavy-tailed claims. They consider also a particular reserve-dependent premium rule, called "adaptive", and describe how to obtain ruin estimates in this setting, both in the infinite and finite horizon case.

\footnotetext{
*This work has been partially supported by Murst Project "Metodi Stocastici in Finanza Matematica".

†Dipartimento di Matematica, Università di Roma "Tor Vergata", Via della Ricerca Scientifica, I-00133 Rome, Italy. e-mail: macci@mat.uniroma2.it

${ }^{\ddagger}$ Istituto per le Applicazioni del Calcolo "Mauro Picone",Consiglio Nazionale delle Ricerche (CNR), c/o Dipartimento di Matematica, Università di Roma "Tor Vergata", Via della Ricerca Scientifica, I-00133 Rome, Italy. e-mail: torrisi@iac.rm.cnr.it
} 
In many real situations, the premium rate is not constant or "adaptive" and it may happen that "induced" and "standard" claims are not identically distributed. For these reasons, here we consider a risk process which differs from the one in Albrecher and Asmussen [AA] for two main aspects: we allow for a not "adaptive" reserve-dependent premium rate; the distributions of "standard" and "induced" claims may be different.

We analyze the proposed model by considering a suitable scaling called slow Markov walk limit (see Cottrell, Fort and Malgouyres [CFT], Bucklew [Bu] and Asmussen and Nielsen [AN]) and we provide asymptotic estimates for ruin probabilities using sample path large deviation techniques. This analysis is carried on under a super-exponential condition on the claim sizes. As typically happens in risk theory, due to the small claim assumption, the ruin probabilities decay to zero exponentially fast. In this paper we solve the related rare event simulation problem providing an asymptotically efficient simulation law for the ruin probabilities by using importance sampling. It is worthwhile to say that, if the claim sizes are not super-exponential (for instance for exponential and heavy tailed claims), the same problems are open and we do not know how to adapt the techniques used in this paper to cope with this case.

In this article we also consider a situation in which the probabilistic structure of the risk process depends on some unknown parameter. In such a case we can have a very misleading inference if we separate the point estimations of the parameter and the ruin probability. Following the lines of some recent works in the literature we can consider a different approach based on Bayesian inference; in this way we provide a wide full statistical analysis capturing all the uncertainty concerning the parameter and the ruin probability, see Ganesh et al. [GGOP] for more insight into this problem. The idea is to consider the so-called predictive ruin probability, i.e. the integral of the ruin probability with respect to the posterior distribution on the unknown parameter. For some technical reasons, the Bayesian estimates for ruin probabilities will be given under the following restrictive assumptions: "standard" and "induced" claims are identically distributed, and their common law is unknown and concentrated on a compact interval. Large deviation estimates for predictive ruin probabilities concerning simpler models can be found in Ganesh and O'Connell [GO1] and [GO2] and in Macci and Petrella [MP]. Among many other references on the Bayesian approach, we cite Ausin and Lopes [AL] and Ausin et al. [AWL] which considered Bayesian estimation of ruin probabilities for different insurance models with heavy tail claim size distributions.

The article is structured as follows. In Section 2 we give a detailed description of the model considered and some useful preliminaries. In Section 3 we show that the scaled risk process satisfies suitable sample path large deviation principles. In Section 4 we study the asymptotic behavior of the ruin probabilities and we address the problem of their Monte Carlo simulation by importance sampling. Finally, in Section 5 we give asymptotic estimates for ruin probabilities in the Bayesian setting. We include two Appendices where we prove some technical details.

Throughout this paper we use the standard notation $[\cdot]$ for the integer part of a real number.

\section{Preliminaries}

\subsection{The model}

In this paper we consider a risk process with premium rate which depends on the current reserve. Risk processes with reserve-dependent premium rate are quite common in the literature: see e.g. Gerber [G], Djehiche [D], Asmussen and Nielsen [AN], Asmussen [A], Ganesh, Macci and Torrisi [GMT].

We consider a claim number process $(N(t))$ given by a Cox process with a Poisson shot noise stochastic intensity (shot noise Cox process). More precisely, let $\lambda, \rho>0$ be positive constants and let $h: \mathbb{R} \times(0, \infty) \rightarrow[0, \infty)$ be a measurable function such that $h(t, y)=0$ for all $t \leq 0$ and 
$y \in(0, \infty)$. The stochastic intensity of $(N(t))$ is

$$
\lambda+\sum_{n=1}^{N^{\rho}(t)} h\left(t-T_{n}, Y_{n}\right)
$$

where: $\left(Y_{n}\right)$ and $\left(N^{\rho}(t)\right)$ are independent, $\left(Y_{n}\right)$ is a sequence of i.i.d. positive random variables, $N^{\rho}(t)=\sum_{n \geq 1} 1_{T_{n} \leq t}$ is a homogeneous Poisson process with intensity $\rho$. In what follows we set $T_{0}=0$

$$
H(t, y)=\int_{-\infty}^{t} h(s, y) d s=\left\{\begin{array}{ll}
\int_{0}^{t} h(s, y) d s & \text { if } t>0 \\
0 & \text { if } t \leq 0
\end{array} \text { and } M_{H(\infty, Y)}(\gamma)=\mathbb{E}\left[e^{\gamma H\left(\infty, Y_{1}\right)}\right]\right.
$$

In view of the definition of the aggregate claims process we consider the following decomposition of $(N(t))$ :

$$
N(t)=N^{\lambda}(t)+\sum_{n=1}^{N^{\rho}(t)} N^{(n)}(t),
$$

where $\left(N^{\lambda}(t)\right)$ is a homogeneous Poisson process with intensity $\lambda$ and each $\left(N^{(n)}\right)$ is a Cox process with intensity $\left(h\left(t-T_{n}, Y_{n}\right)\right)$; moreover the process $N^{\lambda}$ is independent of $N^{\rho}$ and $\left(\left(N^{(n)}\right): n \geq 1\right)$. In particular, for each $n \geq 1, N^{(n)}(\infty)$ is a mixed Poisson random variable with parameter $H\left(\infty, Y_{n}\right)$.

Now we define the aggregate claims process. Let $\left(X_{n}^{\lambda}\right)$ and $\left(X_{i}^{(n)}\right)$ be two independent families of i.i.d. random variables, independent of $(N(t))$. We allow that the common distribution of the random variables $\left(X_{n}^{\lambda}\right)$ and the common distribution of the random variables $\left(X_{i}^{(n)}\right)$ are different; if they coincide we have the same aggregate claims process in [AA]. This generalization allows to model situations where "induced" claims and "standard" claims are not identically distributed. A related model is studied in Yuen et al. [YGN]. We denote the common moment generating function of the random variables $\left(X_{n}^{\lambda}\right)$ by $M_{X^{\lambda}}$, and the common moment generating function of the random variables $\left(X_{i}^{(n)}\right)$ by $M_{X}$, i.e.

$$
M_{X^{\lambda}}(\gamma)=\mathbb{E}\left[e^{\gamma X_{n}^{\lambda}}\right] \text { and } M_{X}(\gamma)=\mathbb{E}\left[e^{\gamma X_{i}^{(n)}}\right] .
$$

Then we define the aggregate claims process as follows:

$$
S(t)=\sum_{n=1}^{N^{\lambda}(t)} X_{n}^{\lambda}+\sum_{n=1}^{N^{\rho}(t)} \sum_{i=1}^{N^{(n)}(t)} X_{i}^{(n)} .
$$

Finally we define the model, i.e. the risk process with reserve dependent premium rate. Let $\left(\mathcal{F}_{t}\right)$ be a complete and right-continuous filtration with respect to which $(S(t))$ is adapted. The process $(S(t))$ has càdlàg and non-decreasing paths; thus it has finite variation on compact sets. Therefore, $(S(t))$ is an $\mathcal{F}_{t}$-semimartingale (see e.g. Protter $[\mathrm{P}]$, Theorem 7, page 47 ). The risk process considered in this paper is the unique strong solution $(R(t))$ of

$$
\left\{\begin{array}{l}
d R(t)=b(R(t)) d t-d S(t) \\
R(0)=u
\end{array}\right.
$$

where we are assuming that $b(\cdot)$ satisfies the following Lipschitz condition:

(L): there exists a constant $L>0$ such that $|b(x)-b(y)| \leq L|x-y|$ for all $x, y \in \mathbb{R}$.

The interpretation in insurance is that $u>0$ is the initial capital, and the nonnegative function $b(\cdot)$ is the premium rate, which depends on the current level of the reserve $(R(t))$.

Note that we meet the risk process studied in Albrecher and Asmussen [AA] when the function $b$ is constant, and the risk process studied in Asmussen and Nielsen [AN] by setting $\rho=0$. On the other hand, to model risk processes with delay in claim settlement and reserve-dependent premium rate, Ganesh, Macci and Torrisi [GMT] considered $(R(t))$ defined by $(2)$ with $(S(t))$ being a Poisson shot noise process. 


\subsection{The batch process and the function $\Lambda$}

Another aggregate claim process $(\widetilde{S}(t))$ plays a crucial role. This aggregate claim process is called batch process (see Albrecher and Asmussen [AA]) and it is obtained from $(S(t))$ as follows: all the claims caused by an occurrence of $\left(N^{\rho}(t)\right)$ at time $T_{n}$ are collected and moved to $T_{n}$.

The batch process is defined by

$$
\widetilde{S}(t)=\sum_{n=1}^{N^{\lambda}(t)} X_{n}^{\lambda}+\sum_{n=1}^{N^{\rho}(t)} \underbrace{\sum_{i=1}^{N^{(n)}(\infty)} X_{i}^{(n)}}_{=: \widehat{X}_{n}} .
$$

We remark that $\left(\widehat{X}_{n}\right)$ is a sequence of i.i.d. random variables, and their (common) moment generating function $M_{\widehat{X}}(\gamma)=\mathbb{E}\left[e^{\gamma \widehat{X}_{n}}\right]$ can be expressed in terms of $M_{X}$ and $M_{H(\infty, Y)}$ defined before:

$$
M_{\widehat{X}}(\gamma)=M_{H(\infty, Y)}\left(M_{X}(\gamma)-1\right)
$$

Moreover, it is important to note that

$$
\widetilde{S}(t) \geq S(t) \text { for all } t \geq 0 .
$$

We now present another useful version of $(\widetilde{S}(t))$ (see Albrecher and Asmussen [AA], Introduction and Subsection 4.4$)$. The process $(\widetilde{S}(t))$ can be written as

$$
\widetilde{S}(t)=\sum_{n=1}^{N^{\lambda+\rho}(t)} \widetilde{X}_{n},
$$

where: $\left(N^{\lambda+\rho}(t)\right)$ is a homogeneous Poisson process with intensity $\lambda+\rho ;\left(\widetilde{X}_{n}\right)$ is a sequence of i.i.d. positive random variables independent of $\left(N^{\lambda+\rho}(t)\right)$; the common distribution of the random variables $\left(\widetilde{X}_{n}\right)$ is a mixture between the distribution of the random variables $\left(X_{n}^{\lambda}\right)$ and the distribution of the random variables $\left(\widehat{X}_{n}\right)$ with weights $\frac{\lambda}{\lambda+\rho}$ and $\frac{\rho}{\lambda+\rho}$, respectively. Thus the (common) moment generating function $M_{\widetilde{X}}(\gamma)=\mathbb{E}\left[e^{\gamma \widetilde{X}_{1}}\right]$ of the random variables $\left(\widetilde{X}_{n}\right)$ is the convex linear combination of $M_{X^{\lambda}}$ and $M_{\widehat{X}}$ with weights $\frac{\lambda}{\lambda+\rho}$ and $\frac{\rho}{\lambda+\rho}$ respectively, i.e. $M_{\widetilde{X}}(\gamma)=$ $\frac{\lambda}{\lambda+\rho} M_{X^{\lambda}}(\gamma)+\frac{\rho}{\lambda+\rho} M_{\widehat{X}}(\gamma)$. Finally, if we consider the function $\Lambda$ defined by

$$
\Lambda(\gamma)=\lambda\left(M_{X^{\lambda}}(\gamma)-1\right)+\rho\left(M_{\widehat{X}}(\gamma)-1\right),
$$

we have

$$
\mathbb{E}\left[e^{\gamma \widetilde{S}(t)}\right]=e^{t(\lambda+\rho)\left(M_{\tilde{X}}(\gamma)-1\right)}=e^{t \Lambda(\gamma)}
$$

\subsection{Preliminaries on large deviations}

The definition of large deviations principle (LDP for short) plays a crucial role in this paper (see Dembo and Zeitouni [DZ] for this definition and further details); here we recall some preliminaries on this topic.

Let $\Omega$ be a topological space with Borel $\sigma$-algebra $\mathcal{B}_{\Omega}$ and call rate function a lower semicontinuous function $I: \Omega \rightarrow[0, \infty]$. Then a family of $\Omega$-valued random variables $\left(Z_{\alpha}\right)$ satisfies the LDP with rate function $I$ if

$$
-\inf _{\omega \in B^{\circ}} I(\omega) \leq \liminf _{\alpha \rightarrow \infty} \frac{1}{\alpha} \log P\left(Z_{\alpha} \in B\right) \leq \limsup _{\alpha \rightarrow \infty} \frac{1}{\alpha} \log P\left(Z_{\alpha} \in B\right) \leq-\inf _{\omega \in \bar{B}} I(\omega)
$$

for all $B \in \mathcal{B}_{\Omega}$. A rate function $I$ is good if all the level sets are compact.

In this paper we use the term sample path large deviations when $\Omega$ is a family of sample paths. More precisely, two classes of families are considered. 
- The first class is $\Omega=D[0, T]$ for some $T \in(0, \infty)$, where $D[0, T]$ is the space of the càdlàg functions on $[0, T]$. In such a case we consider the topology induced by the norm $\|\cdot\|_{[0, T]}$ defined by $\|g\|_{[0, T]}:=\sup _{t \in[0, T]}|g(t)|$, for $g \in D[0, T]$. When we deal with a process $(Z(t))$ (with $t \in[0, T]$ ) having càdlàg paths, we use the notation $(Z(\cdot))_{[0, T]}$.

- The second class is $\Omega=D_{a}^{m}$ for some $a, m \in \mathbb{R}$, where

$$
D_{a}^{m}=\left\{f \in D[0, \infty): f(0)=a, \lim _{t \rightarrow \infty} \frac{f(t)}{1+t}=m\right\}
$$

and $D[0, \infty)$ is the space of the càdlàg functions on $[0, \infty)$. In such a case we consider the topology induced by the metric $d_{a, m}$ defined by

$$
d_{a, m}(f, g)=\sup _{t>0} \frac{|f(t)-g(t)|}{1+t}
$$

(for details see Ganesh, O'Connell and Wischik [GOW], page 154). When we deal with a process $(Z(t))$ (with $t \in[0, \infty)$ ) having càdlàg paths which belong to some $D_{a}^{m}$ with probability 1 , we use the notation $(Z(\cdot))_{[0, \infty)}$.

For the LDP in Section 5 we do not use the term sample path. Indeed, $\Omega$ is the family $M_{1}([0, V])$ of all probability measures on $[0, V]$ (for some $V \in(0, \infty)$ ), equipped with the topology of weak convergence. Furthermore we shall consider a LDP for posterior distributions in Bayesian setting; thus we shall have a "conditional" LDP since the posterior distributions are a random family of probability measures.

\section{Sample path LDPs}

The aim of this section is to prove a suitable sample path LDP for the risk process $(R(t))$ in $(2)$ with a suitable scaling called slow Markov walk limit (see Proposition 3.4 below). A similar result holds for the model with delayed claims studied in Ganesh, Macci and Torrisi [GMT] (see Proposition 3.1 therein).

Let $\Lambda^{*}$ be the Fenchel-Legendre transform of the function $\Lambda$ in (6), i.e.

$$
\Lambda^{*}(x)=\sup _{\gamma \in \mathbb{R}}[\gamma x-\Lambda(\gamma)]
$$

The following condition is needed to prove our results:

(S): $\Lambda(\gamma)<\infty$ for all $\gamma \in \mathbb{R}$.

Condition (S) is a super-exponential condition, i.e. it means that the tails of the random variables $\left(X_{n}^{\lambda}\right)$ and $\left(\widehat{X}_{n}\right)$ go to zero faster than any exponential rate.

\subsection{Sample path large deviations for the aggregate claims process}

We recall that any absolutely continuous function $f$ is almost everywhere differentiable and, from now on, we denote the almost everywhere derivative of $f$ by $\dot{f}$. Let $A C[0, T]$ be the family of all absolutely continuous functions on $[0, T]$ and let $I_{T}$ be the functional defined by

$$
I_{T}(f)= \begin{cases}\int_{0}^{T} \Lambda^{*}(\dot{f}(t)) d t & \text { if } f \in A C[0, T], f(0)=0 \\ \infty & \text { otherwise }\end{cases}
$$

Similarly, let $A C[0, \infty)$ be the family of all absolutely continuous functions on $[0, \infty)$ and let $I_{\infty}$ be the functional defined by

$$
I_{\infty}(f)= \begin{cases}\int_{0}^{\infty} \Lambda^{*}(\dot{f}(t)) d t & \text { if } f \in A C[0, \infty) \cap D_{0}^{\Lambda^{\prime}(0)} \\ \infty & \text { otherwise }\end{cases}
$$


where $\Lambda^{\prime}(0)$ is the derivative of $\Lambda$ at the origin.

In this subsection we prove two sample path LDPs: the LDP of $\left(\left(\frac{S(\alpha \cdot)}{\alpha}\right)_{[0, T]}\right)$ on $D[0, T]$; the LDP of $\left(\left(\frac{S(\alpha \cdot)}{\alpha}\right)_{[0, \infty)}\right)$ on $D_{0}^{\Lambda^{\prime}(0)}$. We first recall the following well-known result due to Borovkov $[\mathrm{Bo}]$ (see also de Acosta [d] and the references cited therein).

Proposition 3.1 Assume $(S)$. Then $\left(\left(\frac{\widetilde{S}(\alpha \cdot)}{\alpha}\right)_{[0, T]}\right)$ satisfies the LDP with good rate function $I_{T}$.

The following proposition holds.

Proposition 3.2 Assume $(\boldsymbol{S})$. Then $\left(\left(\frac{S(\alpha \cdot)}{\alpha}\right)_{[0, T]}\right)$ satisfies the LDP with good rate function $I_{T}$.

Proof. Proposition 3.1 provides the LDP of $\left(\left(\frac{\widetilde{S}(\alpha \cdot)}{\alpha}\right)_{[0, T]}\right)$ and the goodness of the rate function $I_{T}$. Thus, by Theorem 4.2.13 in Dembo and Zeitouni [DZ], we only need to show that $\left(\left(\frac{S(\alpha \cdot)}{\alpha}\right)_{[0, T]}\right)$ and $\left(\left(\frac{\widetilde{S}(\alpha \cdot)}{\alpha}\right)_{[0, T]}\right)$ are exponentially equivalent as $\alpha \rightarrow \infty$, i.e.

$$
\lim _{\alpha \rightarrow \infty} \frac{1}{\alpha} \log P\left(\frac{1}{\alpha} \sup _{t \in[0, T]}|\widetilde{S}(\alpha t)-S(\alpha t)|>\delta\right)=-\infty(\text { for all } \delta>0) ;
$$

see Appendix A for the measurability requirement concerning the events in (7) (varying $\alpha>0$ ).

By (1), (3) and (4) we have

$$
\sup _{t \in[0, T]}|\widetilde{S}(\alpha t)-S(\alpha t)|=\sup _{t \in[0, T]} \sum_{n=1}^{N^{\rho}(t)} \sum_{i=1}^{N^{(n)}(\infty)} X_{i}^{(n)}-\sum_{n=1}^{N^{\rho}(t)} \sum_{i=1}^{N^{(n)}(\alpha t)} X_{i}^{(n)} .
$$

Note that, when $t \in\left[\frac{T_{m-1}}{\alpha}, \frac{T_{m}}{\alpha}\right)$ for some $m$, we have:

$$
\begin{aligned}
& \sum_{n=1}^{N^{\rho}(t)} \sum_{i=1}^{N^{(n)}(\infty)} X_{i}^{(n)} \text { is constant w.r.t. } t \\
& \sum_{n=1}^{N^{\rho}(t)} \sum_{i=1}^{N^{(n)}(\alpha t)} X_{i}^{(n)} \text { is nondecreasing w.r.t. } t .
\end{aligned}
$$

Thus, $\sup _{t \in[0, T]}|\widetilde{S}(\alpha t)-S(\alpha t)|$ is attained at $t=\frac{T_{m}}{\alpha} \in[0, T]$ for some $m$ :

$$
\begin{gathered}
\sup _{t \in[0, T]}|\widetilde{S}(\alpha t)-S(\alpha t)|=\sup _{m: \frac{T_{m}}{\alpha} \in[0, T]} \sum_{n=1}^{m} \sum_{i=1}^{N^{(n)}(\infty)} X_{i}^{(n)}-\sum_{n=1}^{m} \sum_{i=1}^{N^{(n)}\left(T_{m}\right)} X_{i}^{(n)}= \\
=\sup _{m: T_{m} \leq \alpha T} \sum_{n=1}^{m}\left(\sum_{i=1}^{N^{(n)}(\infty)} X_{i}^{(n)}-\sum_{i=1}^{N^{(n)}\left(T_{m}\right)} X_{i}^{(n)}\right) .
\end{gathered}
$$

We remark that the processes $\left(N^{(1)}\left(t+T_{1}\right)\right), \ldots,\left(N^{(m)}\left(t+T_{m}\right)\right)$ are i.i.d., and independent of $\left(N^{\rho}(t)\right)$; in what follows we use the notation

$$
N_{m-n}^{*}(t)=N^{(n)}\left(t+T_{n}\right)(\text { for } n \in\{1, \ldots, m\} \text { and } t \geq 0) .
$$

Then, by taking into account that

$$
\left(T_{m}-T_{1}, \ldots, T_{m}-T_{m-1}, T_{m}-T_{m}\right)=^{d}\left(T_{m-1}, \ldots, T_{m-(m-1)}, T_{m-m}\right)
$$

(where, from now on, $={ }^{d}$ means "equally distributed"), we have

$$
\sup _{t \in[0, T]}|\widetilde{S}(\alpha t)-S(\alpha t)|={ }^{d} \sup _{m: T_{m} \leq \alpha T} \sum_{n=1}^{m}\left(\sum_{i=1}^{N_{m-n}^{*}(\infty)} X_{i}^{(m-n+1)}-\sum_{i=1}^{N_{m-n}^{*}\left(T_{m-n}\right)} X_{i}^{(m-n+1)}\right) .
$$


Then, if we consider the random variables $\left(A_{m}\right)$ defined by

$$
A_{m}=\sum_{j=0}^{m-1}\left(\sum_{i=1}^{N_{j}^{*}(\infty)} X_{i}^{(j+1)}-\sum_{i=1}^{N_{j}^{*}\left(T_{j}\right)} X_{i}^{(j+1)}\right),
$$

we have

$$
\sup _{t \in[0, T]}|\widetilde{S}(\alpha t)-S(\alpha t)|={ }^{d} \sup _{m: T_{m} \leq \alpha T} A_{m}=A_{N \rho(\alpha T)}
$$

since $\left(A_{m}\right)$ is a nondecreasing sequence (being the sequence of partial sums of nonnegative random variables).

Therefore (7) is equivalent to

$$
\lim _{\alpha \rightarrow \infty} \frac{1}{\alpha} \log P\left(A_{N^{\rho}(\alpha T)}>\alpha \delta\right)=-\infty(\text { for all } \delta>0) .
$$

The following estimates (9) and (11) are needed to check (8).

- Let $\eta>0$ and a positive integer $K$ be arbitrarily fixed. Since $T_{n}$ is the sum of $n$ independent exponential random variables with mean $\frac{1}{\rho}$, we have

$$
P\left(T_{K[\alpha]}<\alpha T\right) \leq e^{\eta \alpha T} \mathbb{E}\left[e^{\left.-\eta T_{K[\alpha]}\right]}=e^{\eta \alpha T}\left(\frac{\rho}{\rho+\eta}\right)^{K[\alpha]},\right.
$$

and therefore

$$
\lim _{\alpha \rightarrow \infty} \frac{1}{\alpha} \log P\left(T_{K[\alpha]}<\alpha T\right) \leq \eta T+K \log \frac{\rho}{\rho+\eta} .
$$

- Let $\theta, \delta>0$ and a positive integer $K$ be arbitrarily fixed. We have

$$
P\left(A_{N^{\rho}(\alpha T)}>\alpha \delta, T_{K[\alpha]} \geq \alpha T\right) \leq P\left(A_{K[\alpha]} \geq \alpha \delta\right) \leq e^{-\theta \alpha \delta} \mathbb{E}\left[e^{\left.\theta A_{K[\alpha]}\right]} ;\right.
$$

thus, since we have

$$
\left.\lim _{m \rightarrow \infty} \frac{1}{m} \log \mathbb{E}\left[e^{\theta A_{m}}\right]=0 \text { (for all } \theta>0\right)
$$

(the proof of (10) is given in the Appendix B), we obtain

$$
\limsup _{\alpha \rightarrow \infty} \frac{1}{\alpha} \log P\left(A_{N^{\rho}(\alpha T)}>\alpha \delta, T_{K[\alpha]} \geq \alpha T\right) \leq-\theta \delta .
$$

Therefore

$$
\lim _{\alpha \rightarrow \infty} \frac{1}{\alpha} \log P\left(A_{N^{\rho}(\alpha T)}>\alpha \delta, T_{K[\alpha]} \geq \alpha T\right)=-\infty
$$

since $\theta>0$ is arbitrary.

By the union bound we get

$$
P\left(A_{N^{\rho}(\alpha T)}>\alpha \delta\right) \leq P\left(A_{N^{\rho}(\alpha T)}>\alpha \delta, T_{K[\alpha]} \geq \alpha T\right)+P\left(T_{K[\alpha]}<\alpha T\right) ;
$$

hence, by (9) and (11), we have

$$
\limsup _{\alpha \rightarrow \infty} \frac{1}{\alpha} \log P\left(A_{N^{\rho}(\alpha T)}>\alpha \delta\right) \leq \eta T+K \log \frac{\rho}{\rho+\eta} .
$$

Moreover, for $K>\rho T$, we can set $\eta=\frac{K}{T}-\rho$ and we have

$$
\limsup _{\alpha \rightarrow \infty} \frac{1}{\alpha} \log P\left(A_{N^{\rho}(\alpha T)}>\alpha \delta\right) \leq K-\rho T-K \log \frac{K}{\rho T} .
$$


Finally (8) follows by letting $K$ go to infinity.

A consequence of Proposition 3.2 is that $\lim _{t \rightarrow \infty} \frac{S(t)}{t}=\Lambda^{\prime}(0)$ a.s.. Note also that

$$
\begin{aligned}
& \Lambda^{\prime}(0)=\lambda M_{X^{\lambda}}^{\prime}(0)+\rho M_{\widehat{X}}^{\prime}(0)=\lambda M_{X^{\lambda}}^{\prime}(0)+\rho M_{H(\infty, Y)}^{\prime}(0) M_{X}^{\prime}(0), \\
& \text { i.e. } \Lambda^{\prime}(0)=\lambda \mathbb{E}\left[X_{1}^{\lambda}\right]+\rho \mathbb{E}\left[H\left(\infty, Y_{1}\right) \mathbb{E}\left[X_{1}\right]\right.
\end{aligned}
$$

Proposition 3.3 Assume $(\boldsymbol{S})$. Then $\left(\left(\frac{S(\alpha \cdot)}{\alpha}\right)_{[0, \infty)}\right)$ satisfies the LDP in $D_{0}^{\Lambda^{\prime}(0)}$ with good rate function $I_{\infty}$.

Proof. By Theorem 18 and Lemma 19 in Majewski $[\mathrm{M}]$ we only have to check the following condition: for every $\varepsilon, \mu>0$ there exist $\tau>0$ and $c \in \mathbb{R}$ such that

$$
\limsup _{\alpha \rightarrow \infty} \frac{1}{\alpha} \log P\left(\sup _{t \in[\tau, 2 \tau]}\left|\frac{S(\alpha t)}{\alpha t}-c\right| \geq \varepsilon\right)<-\mu .
$$

In what follows we show that this condition holds choosing $c=\Lambda^{\prime}(0)$ and $\tau>\frac{\mu}{\min \left\{\Lambda^{*}\left(\Lambda^{\prime}(0)-\varepsilon\right), \Lambda^{*}\left(\Lambda^{\prime}(0)+\varepsilon\right)\right\}}$ for any given $\varepsilon, \mu>0$. Firstly, by Proposition 3.2 (with $T=2 \tau$ ), we have

$$
\limsup _{\alpha \rightarrow \infty} \frac{1}{\alpha} \log P\left(\sup _{t \in[\tau, 2 \tau]}\left|\frac{S(\alpha t)}{\alpha t}-c\right| \geq \varepsilon\right) \leq-\inf \left\{I_{2 \tau}(f): f \in E\right\}
$$

where

$$
E=\left\{f \in A C[0,2 \tau]: f(0)=0, \sup _{t \in[\tau, 2 \tau]}\left|\frac{f(t)}{t}-c\right| \geq \varepsilon\right\} .
$$

Moreover let $\widetilde{E}$ be the set of functions $g=g_{s, x}$ such that

$$
g_{s, x}(t)= \begin{cases}x t & \text { if } t \in[0, s] \\ x s+\Lambda^{\prime}(0)(t-s) & \text { if } t \in(s, 2 \tau]\end{cases}
$$

for some $(s, x)$ such that $s \in[\tau, 2 \tau]$ and $|x-c| \geq \varepsilon$. Then, since for all $f \in E$ there exists $s \in[\tau, 2 \tau]$ such that $\left|\frac{f(s)}{s}-c\right| \geq \varepsilon$, we have

$$
I_{2 \tau}(f)=\int_{0}^{2 \tau} \Lambda^{*}(\dot{f}(t)) d t \geq \int_{0}^{s} \Lambda^{*}(\dot{f}(t)) d t \geq s \Lambda^{*}(f(s) / s)=I_{2 \tau}\left(g_{s, f(s) / s}\right) ;
$$

combining this with (12) we get

$$
\limsup _{\alpha \rightarrow \infty} \frac{1}{\alpha} \log P\left(\sup _{t \in[\tau, 2 \tau]}\left|\frac{S(\alpha t)}{\alpha t}-c\right| \geq \varepsilon\right) \leq-\inf \left\{I_{2 \tau}(f): f \in \widetilde{E}\right\} .
$$

We complete the proof noting that

$$
\begin{gathered}
\inf \left\{I_{2 \tau}(f): f \in \widetilde{E}\right\}=\inf \left\{s \Lambda^{*}(x): s \in[\tau, 2 \tau],|x-c| \geq \varepsilon\right\}= \\
=\tau \min \left\{\Lambda^{*}\left(\Lambda^{\prime}(0)-\varepsilon\right), \Lambda^{*}\left(\Lambda^{\prime}(0)+\varepsilon\right)\right\}<-\mu .
\end{gathered}
$$




\subsection{Sample path large deviations for the reserve process}

Now we introduce a suitable scaling called slow Markov walk limit. More in particular, for each fixed $\alpha>0$, we consider the reserve process $\left(R^{\alpha}(t)\right)$ defined by

$$
\left\{\begin{array}{l}
d R^{\alpha}(t)=b\left(R^{\alpha}(t)\right) d t-\frac{1}{\alpha} \cdot d S(\alpha t) \\
R^{\alpha}(0)=u
\end{array} .\right.
$$

Now we introduce some further assumptions and notations.

(A): There exists $B>0$ such that $\lim _{x \rightarrow \infty} b(x)=B$.

(N): For $\underline{b}:=\inf _{x \in \mathbb{R}} b(x)$, we have $\underline{b}>\Lambda^{\prime}(0)$.

We remark that, if $(\mathbf{A})$ and $(\mathbf{N})$ hold, we have $B>\Lambda^{\prime}(0)$. Condition (A) gives the asymptotic behavior of $b(\cdot)$ and $(\mathbf{N})$ plays the role of the net profit condition in our context.

In this subsection we state the LDP of $\left(\left(R^{\alpha}(\cdot)\right)_{[0, \infty)}\right)$ on $D_{u}^{B-\Lambda^{\prime}(0)}$. We remark that, assuming $(\mathbf{L}),(\mathbf{S}),(\mathbf{A})$ and $(\mathbf{N})$, we can easily check that the random paths $\left(\left(R^{\alpha}(\cdot)\right)_{[0, \infty)}\right)$ are almost surely elements of $D_{u}^{B-\Lambda^{\prime}(0)}$. Indeed, we have $\liminf _{t \rightarrow \infty} \frac{R^{\alpha}(t)}{1+t} \geq \underline{b}-\Lambda^{\prime}(0)>0$ by $(13), \lim _{t \rightarrow \infty} \frac{S(t)}{t}=\Lambda^{\prime}(0)$ and $(\mathbf{N})$; thus $\lim _{t \rightarrow \infty} R^{\alpha}(t)=\infty$, and therefore $\lim _{t \rightarrow \infty} \frac{R^{\alpha}(t)}{1+t}=B-\Lambda^{\prime}(0)>0$. In view of the next result, consider the rate function $J$ defined by

$$
J(g)=\left\{\begin{array}{ll}
\int_{0}^{\infty} \Lambda^{*}(-\dot{g}(t)+b(g(t))) d t & \text { if } g \in A C[0, \infty) \cap D_{u}^{B-\Lambda^{\prime}(0)} \\
\infty & \text { otherwise }
\end{array} .\right.
$$

The following proposition holds.

Proposition 3.4 Assume $(\boldsymbol{L}),(\boldsymbol{S}),(\boldsymbol{A})$ and $(\boldsymbol{N})$. Then $\left(\left(R^{\alpha}(\cdot)\right)_{[0, \infty)}\right)$ satisfies the LDP with good rate function $J$.

Proof. It is an easy consequence of Proposition 3.3, and it is similar to the proof of Proposition 3.1 of Ganesh, Macci and Torrisi [GMT].

\section{Ruin probabilities}

The aim of this section is to present asymptotic estimates for the ruin probabilities concerning the risk processes $\left(R^{\alpha}(\cdot)\right)$ defined by (13) (see Proposition 4.1 below). Moreover, we provide an asymptotically efficient simulation law for the ruin probabilities by using importance sampling (see Proposition 4.3 below). Similar results for the models with undelayed and delayed claims can be found in Asmussen and Nielsen [AN] (see Corollaries 1 and 2 therein) and in Ganesh, Macci and Torrisi [GMT] (see Propositions 4.2 and 5.2 therein), respectively.

\subsection{Asymptotic behavior}

In this subsection we provide the asymptotic behavior of the ruin probabilities. We start with some preliminaries. Since the claims $\left(X_{n}\right)$ are positive, conditions $(\mathbf{S})$ and $(\mathbf{N})$ imply the following condition.

(C): For all $c \geq \underline{b}$ there exists a unique $\gamma_{c}>0$ such that $\Lambda\left(\gamma_{c}\right)-c \gamma_{c}=0$.

The values $\left(\gamma_{c}: c \geq \underline{b}\right)$ are called local adjustment coefficients. Furthermore, when (C) holds, for all $c \geq \underline{b}$ we have

$$
\inf _{t>0} t \Lambda^{*}\left(c+\frac{1}{t}\right)=\frac{\Lambda^{*}\left(\Lambda^{\prime}\left(\gamma_{c}\right)\right)}{\Lambda^{\prime}\left(\gamma_{c}\right)-c}=\gamma_{c}
$$

more precisely the infimum is attained at $t=\frac{1}{\Lambda^{\prime}\left(\gamma_{c}\right)-c}$ and this minimizer is unique, since we have a strictly convex function of $t$ by the strict convexity of $\Lambda^{*}$. 
Define the (infinite horizon) ruin probability

$$
\Psi_{\alpha}(u)=P\left(\exists t>0: R^{\alpha}(t) \leq 0 \mid R^{\alpha}(0)=u\right)
$$

and the function

$$
w(u)=\inf \left\{J(g): g \in \mathcal{S}^{u}\right\},
$$

where

$$
\mathcal{S}^{u}=\{g \in A C[0, \infty): g(0)=u, g(t)=0 \text { for some } t>0\} .
$$

The following proposition holds.

Proposition 4.1 Assume the hypotheses of Proposition 3.4. Then, for each $u>0$ arbitrarily fixed, we have

$$
\lim _{\alpha \rightarrow \infty} \frac{1}{\alpha} \log \Psi_{\alpha}(u)=-w(u)=-\int_{0}^{u} \gamma_{b(x)} d x .
$$

Proof. Due to the LDP in Proposition 3.4, we can adapt the proof of Propositions 4.1 and 4.2 of Ganesh, Macci and Torrisi [GMT].

\subsection{Monte Carlo simulation of ruin probabilities}

In this subsection we address the problem of estimation of $\Psi_{\alpha}(u)$ (as $\alpha \rightarrow \infty$ ) by a Monte Carlo simulation. More precisely, we determine an asymptotically efficient simulation law for $\Psi_{\alpha}(u)$, as $\alpha \rightarrow \infty$. To this end, we use importance sampling (see for instance Bucklew [Bu]).

We consider the following conditions.

(R): There exists $t_{\max } \in(0, \infty)$ such that, for all $y \in(0, \infty)$, we have $H\left(t_{\max }, y\right)=H(\infty, y)=y$. (M): The function $b(\cdot)$ is non-decreasing, and $B=\sup _{x \in \mathbb{R}} b(x)<\Lambda^{\prime}\left(\gamma_{\underline{b}}\right)$.

For instance, condition (R) holds if $H(t, y)=H(t) y$ where $H(t)$ is the distribution function of a bounded and nonnegative random variable, i.e. $H(0)=0$ and $H\left(t_{\max }\right)=1$ for some $t_{\max } \in(0, \infty)$. The monotonicity of $b(\cdot)$ in (M) also appears in Asmussen and Nielsen [AN] and in Ganesh, Macci and Torrisi [GMT].

We start recalling some preliminaries on importance sampling, and then we present a suitable asymptotically efficient simulation law $\bar{Q}$.

Preliminaries on importance sampling. We can write the ruin probability $\Psi_{\alpha}(u)$ in terms of the ruin time $\tau_{u}^{\alpha}$ as follows:

$$
\Psi_{\alpha}(u)=P\left(\tau_{u}^{\alpha}<\infty\right), \text { where } \tau_{u}^{\alpha}=\inf \left\{t \geq 0: R^{\alpha}(t) \leq 0\right\} .
$$

Consider $r$ independent replications of $\tau_{u}^{\alpha}$ under the original law $P$, say $\tau_{u, 1}^{\alpha}, \ldots, \tau_{u, r}^{\alpha}$. The corresponding crude Monte Carlo estimator of $\Psi_{\alpha}(u)$ is

$$
\widehat{\Psi_{\alpha}(u)}=\frac{1}{r} \sum_{i=1}^{r} \mathbf{1}_{\tau_{u, i}^{\alpha}<\infty} .
$$

Its relative error is

$$
\frac{1}{\Psi_{\alpha}(u)} \sqrt{\frac{\Psi_{\alpha}(u)\left(1-\Psi_{\alpha}(u)\right)}{r}}
$$

thus, by Proposition 4.1, $r$ needs to grow exponentially with $\alpha$, as $\alpha \rightarrow \infty$, to keep a fixed relative error. This makes the crude Monte Carlo estimator not efficient to estimate $\Psi_{\alpha}(u)$, as $\alpha \rightarrow \infty$.

To overcome this problem, the idea is to consider $r$ independent replications of $\tau_{u}^{\alpha}$ under another law $Q$ on the underlying measurable space $(\Omega, \mathcal{F})$. From now on we set $\mathcal{F}_{t}^{\alpha}:=\mathcal{F}_{\alpha t}$ for all $\alpha>0$ and 
$t \geq 0$. We choose $Q$ such that: $Q$ is absolutely continuous with respect to the original law $P$ on $\mathcal{F}_{t}^{\alpha}$ with a strictly positive density $\frac{d Q_{t}^{\alpha}}{d P_{t}^{\alpha}} ; Q$ is admissible for simulations, i.e. $Q\left(\tau_{u}^{\alpha}<\infty\right)=1$ (for all $\alpha>0)$. The corresponding importance sampling estimator $\left[\widehat{\Psi_{\alpha}(u)}\right]_{Q}$ of $\Psi_{\alpha}(u)$ is defined by

$$
\left[\widehat{\Psi_{\alpha}(u)}\right]_{Q}=\frac{1}{r} \sum_{i=1}^{r} \frac{d P_{\tau_{u, i}^{\alpha}}^{\alpha}}{d Q_{\tau_{u, i}^{\alpha}}^{\alpha}} \mathbf{1}_{\tau_{u, i}^{\alpha}<\infty}
$$

and it is unbiased. Its variance is

$$
\left.\operatorname{Var}_{Q}\left[\widehat{\Psi_{\alpha}(u)}\right]_{Q}\right]=\frac{\mathbb{E}_{Q}\left[\left(\frac{d P_{\tau_{u}^{\alpha}}^{\alpha}}{d Q_{\tau_{u}^{\alpha}}^{\alpha}}\right)^{2} \mathbf{1}_{\tau_{u}^{\alpha}<\infty}\right]-\left(\Psi_{\alpha}(u)\right)^{2}}{r}=\frac{\mathbb{E}_{Q}\left[\left(\frac{d P_{\tau_{u}^{\alpha}}^{\alpha}}{d Q_{\tau_{u}^{\alpha}}^{\alpha}}\right)^{2}\right]-\left(\Psi_{\alpha}(u)\right)^{2}}{r},
$$

where the second equality holds since $Q\left(\tau_{u}^{\alpha}<\infty\right)=1$; we remark that the second moment

$$
\eta_{Q}(\alpha)=\mathbb{E}_{Q}\left[\left(\frac{d P_{\tau_{u}^{\alpha}}^{\alpha}}{d Q_{\tau_{u}^{\alpha}}^{\alpha}}\right)^{2}\right]
$$

is the only part of the variance which depends on $Q$.

The minimization of the variance (or equivalently of the second moment) is often intractable for a fixed $\alpha$. Thus, in order to apply the large deviation estimates in this paper, we look for an asymptotic minimization as $\alpha \rightarrow \infty$. By taking into account Proposition 4.1 and following Siegmund's criterion (see Siegmund [S]; see also Lehtonen and Nyrhinen [LN]), we say that an admissible law $Q$ is asymptotically efficient, as $\alpha \rightarrow \infty$, if

$$
\lim _{\alpha \rightarrow \infty} \frac{1}{\alpha} \log \eta_{Q}(\alpha)=-2 \int_{0}^{u} \gamma_{b(x)} d x .
$$

The law $\bar{Q}$. Let $\left(\widetilde{R}^{\alpha}(t)\right)$ be the risk process defined by

$$
\left\{\begin{array}{l}
d \widetilde{R}^{\alpha}(t)=b\left(\widetilde{R}^{\alpha}(t)\right) d t-\frac{1}{\alpha} \cdot d \widetilde{S}(\alpha t) \\
\widetilde{R}^{\alpha}(0)=u
\end{array}\right.
$$

where $(\widetilde{S}(t))$ is the batch process. We define the law $\bar{Q}$ by a suitable exponential tilting: for each fixed $\alpha>0$ and $t \geq 0$, the law $\bar{Q}$ is absolutely continuous with respect to $P$ on $\mathcal{F}_{t}^{\alpha}$ with density

$$
\frac{d \bar{Q}_{t}^{\alpha}}{d P_{t}^{\alpha}}=\exp \left(-\alpha \int_{0}^{t} \gamma_{b\left(\widetilde{R}^{\alpha}(s-)\right)} d \widetilde{R}^{\alpha}(s)\right)
$$

As pointed out by Asmussen and Nielsen [AN] (see Proposition 3 therein), $\bar{Q}$ makes $\widetilde{R}^{\alpha}$ a suitable risk process such that the premium rate is $b(\cdot)$ and the items concerning $\left(\frac{\widetilde{S}(\alpha t)}{\alpha}\right)$ depend on the current level of the reserve $\widetilde{R}^{\alpha}$. We consider two presentations of these items which depend on the versions $(5)$ and $(3)$ of $(\widetilde{S}(t))$.

We start considering $(\widetilde{S}(t))$ in $(5)$. Under $\bar{Q}$, if $\widetilde{R}^{\alpha}(t)=x$, we have that: the arrival rate of a claim $\frac{\tilde{X}}{\alpha}$ is $(\lambda+\rho)_{x}^{(\alpha)}=\alpha(\lambda+\rho) M_{\widetilde{X}}\left(\gamma_{b(x)}\right)$; the law $\bar{Q}_{\tilde{X} / \alpha}^{(x)}$ of a claim $\frac{\widetilde{X}}{\alpha}$ is $d \bar{Q}_{\tilde{X} / \alpha}^{(x)}(z)=$ $\frac{e^{\alpha \gamma_{b(x)} z}}{M_{\tilde{X}}\left(\gamma_{b(x)}\right)} d P_{\tilde{X} / \alpha}(z)$, where $P_{\widetilde{X} / \alpha}$ is the common law of the random variables $\left(\frac{\widetilde{X}_{n}}{\alpha}\right)$ under $P$.

If we consider $(\widetilde{S}(t))$ in $(3)$, some further computations are needed. In view of this we denote the common law of the random variables $\left(\frac{X_{n}^{\lambda}}{\alpha}\right)$ (under the original law $P$ ) by $P_{X^{\lambda} / \alpha}$; similarly we denote the common law of the random variables $\left(\frac{\widehat{X}_{n}}{\alpha}\right)$ (again under $P$ ) by $P_{\widehat{X} / \alpha}$. Then we have

$$
(\lambda+\rho)_{x}^{(\alpha)}=\alpha(\lambda+\rho) M_{\widetilde{X}}\left(\gamma_{b(x)}\right)=
$$




$$
=\alpha(\lambda+\rho)\left[\frac{\lambda}{\lambda+\rho} M_{X^{\lambda}}\left(\gamma_{b(x)}\right)+\frac{\rho}{\lambda+\rho} M_{\widehat{X}}\left(\gamma_{b(x)}\right)\right]=\alpha \lambda M_{X^{\lambda}}\left(\gamma_{b(x)}\right)+\alpha \rho M_{\widehat{X}}\left(\gamma_{b(x)}\right),
$$

and

$$
\begin{gathered}
d \bar{Q}_{\tilde{X} / \alpha}^{(x)}(z)=\frac{e^{\alpha \gamma_{b(x)} z}}{M_{\widetilde{X}}\left(\gamma_{b(x)}\right)} d P_{\widetilde{X} / \alpha}(z)= \\
=\frac{e^{\alpha \gamma_{b(x)} z}}{\frac{\lambda}{\lambda+\rho} M_{X^{\lambda}}\left(\gamma_{b(x)}\right)+\frac{\rho}{\lambda+\rho} M_{\widehat{X}}\left(\gamma_{b(x)}\right)}\left[\frac{\lambda}{\lambda+\rho} d P_{X^{\lambda} / \alpha}(z)+\frac{\rho}{\lambda+\rho} d P_{\widehat{X} / \alpha}(z)\right]= \\
=\frac{\lambda e^{\alpha \gamma_{b(x)} z} d P_{X^{\lambda} / \alpha}(z)+\rho e^{\alpha \gamma_{b(x)} z} d P_{\widehat{X} / \alpha}(z)}{\lambda M_{X^{\lambda}}\left(\gamma_{b(x)}\right)+\rho M_{\widehat{X}}\left(\gamma_{b(x)}\right)}= \\
=\frac{\lambda M_{X^{\lambda}}\left(\gamma_{b(x)}\right) \frac{e^{\alpha \gamma_{b(x)} z}}{M_{X^{\lambda}\left(\gamma_{b(x)}\right)}} d P_{X^{\lambda} / \alpha}(z)+\rho M_{\widehat{X}}\left(\gamma_{b(x)}\right) \frac{e^{\alpha \gamma_{b(x)} z}}{M_{\widehat{X}}\left(\gamma_{b(x)}\right)} d P_{\widehat{X} / \alpha}(z)}{\lambda M_{X^{\lambda}}\left(\gamma_{b(x)}\right)+\rho M_{\widehat{X}}\left(\gamma_{b(x)}\right)} .
\end{gathered}
$$

Then, under $\bar{Q}$, if $\widetilde{R}^{\alpha}(t)=x$, we have that: the arrival rate of a claim $\frac{X^{\lambda}}{\alpha}$ is $\lambda_{x}^{(\alpha)}=\alpha \lambda M_{X^{\lambda}}\left(\gamma_{b(x)}\right)$; the law $\bar{Q}_{X^{\lambda} / \alpha}^{(x)}$ of a claim $\frac{X^{\lambda}}{\alpha}$ is $d \bar{Q}_{X^{\lambda} / \alpha}^{(x)}(z)=\frac{e^{\alpha \gamma_{b(x)} z}}{M_{X^{\lambda}}\left(\gamma_{b(x)}\right)} d P_{X^{\lambda} / \alpha}(z)$; the arrival rate of a claim $\frac{\widehat{X}}{\alpha}$ is $\rho_{x}^{(\alpha)}=\alpha \rho M_{\widehat{X}}\left(\gamma_{b(x)}\right)$; the law $\bar{Q}_{\widehat{X} / \alpha}^{(x)}$ of a claim $\frac{\widehat{X}}{\alpha}$ is $d \bar{Q}_{\widehat{X} / \alpha}^{(x)}(z)=\frac{e^{\alpha \gamma_{b(x)} z}}{M_{\widehat{X}}\left(\gamma_{b(x)}\right)} d P_{\widehat{X} / \alpha}(z)$.

Some further details can be given. Let $(\mu)^{* j}$ be the $j$-fold convolution of a law $\mu$, let $P_{Y}$ be the common law of the random variables $\left(Y_{n}\right)$ under $P$ and let $\bar{Q}_{N}^{(x)}(h)$ be the discrete density (on nonnegative integers $h$ ) defined by

$$
\bar{Q}_{N}^{(x)}(h)=\frac{\int_{0}^{\infty} \frac{\left(M_{X}\left(\gamma_{b(x)}\right) y\right)^{h}}{h !} e^{-y} d P_{Y}(y)}{\sum_{j=0}^{\infty} \int_{0}^{\infty} \frac{\left(M_{X}\left(\gamma_{b(x)}\right) y\right)^{j}}{j !} e^{-y} d P_{Y}(y)} .
$$

We remark that

$$
\bar{Q}_{N}^{(x)}(h)=\frac{P\left(N^{(1)}(\infty)=h\right) M_{X}^{h}\left(\gamma_{b(x)}\right)}{M_{\widehat{X}}\left(\gamma_{b(x)}\right)} ;
$$

indeed

$$
\int_{0}^{\infty} \frac{\left(M_{X}\left(\gamma_{b(x)}\right) y\right)^{h}}{h !} e^{-y} d P_{Y}(y)=\int_{0}^{\infty} \frac{y^{h}}{h !} e^{-y} d P_{Y}(y) M_{X}^{h}\left(\gamma_{b(x)}\right)=P\left(N^{(1)}(\infty)=h\right) M_{X}^{h}\left(\gamma_{b(x)}\right),
$$

whence we obtain

$$
\sum_{j=0}^{\infty} \int_{0}^{\infty} \frac{\left(M_{X}\left(\gamma_{b(x)}\right) y\right)^{j}}{j !} e^{-y} d P_{Y}(y)=\sum_{j=0}^{\infty} P\left(N^{(1)}(\infty)=j\right) M_{X}^{j}\left(\gamma_{b(x)}\right)=M_{\widehat{X}}\left(\gamma_{b(x)}\right) .
$$

Then we have

$$
\begin{aligned}
& d \bar{Q}_{\widehat{X} / \alpha}^{(x)}(z)=\frac{e^{\alpha \gamma_{b(x)} z}}{M_{\widehat{X}}\left(\gamma_{b(x)}\right)} d P_{\widehat{X} / \alpha}(z)=\frac{e^{\alpha \gamma_{b(x)} z}}{M_{\widehat{X}}\left(\gamma_{b(x)}\right)} \sum_{j=0}^{\infty} P\left(N^{(1)}(\infty)=j\right) d\left(P_{X / \alpha}\right)^{* j}(z)= \\
= & \sum_{j=0}^{\infty} \frac{P\left(N^{(1)}(\infty)=j\right) M_{X}^{j}\left(\gamma_{b(x)}\right)}{M_{\widehat{X}}\left(\gamma_{b(x)}\right)} \frac{e^{\alpha \gamma_{b(x)} z}}{M_{X}^{j}\left(\gamma_{b(x)}\right)} d\left(P_{X / \alpha}\right)^{* j}(z)=\sum_{j=0}^{\infty} \bar{Q}_{N}^{(x)}(j) d\left(\bar{Q}_{X / \alpha}^{(x)}\right)^{* j}(z) ;
\end{aligned}
$$

moreover, since $\bar{Q}_{N}^{(x)}(h)=\frac{\int_{0}^{\infty} \frac{\left(M_{X}\left(\gamma_{b(x)}\right) y\right)^{h}}{h !} e^{-y} d P_{Y}(y)}{M_{\hat{X}}\left(\gamma_{b(x)}\right)}$, we obtain

$$
\bar{Q}_{N}^{(x)}(h)=\int_{0}^{\infty} \frac{\left(M_{X}\left(\gamma_{b(x)}\right) y\right)^{h}}{h !} e^{-M_{X}\left(\gamma_{b(x)}\right) y} \frac{e^{\left\{M_{X}\left(\gamma_{b(x)}\right)-1\right\} y}}{M_{\widehat{X}}\left(\gamma_{b(x)}\right)} d P_{Y}(y) ;
$$


therefore, under $\bar{Q}$, if $\widetilde{R}^{\alpha}(t)=x$, the random variable $N(\infty)$ is mixed Poisson distributed with intensity $M_{X}\left(\gamma_{b(x)}\right) Y$, where the random variable $Y$ has distribution $\bar{Q}_{Y}^{(x)}$ defined by

$$
d \bar{Q}_{Y}^{(x)}(y)=\frac{e^{\left\{M_{X}\left(\gamma_{b(x)}\right)-1\right\} y}}{M_{\widehat{X}}\left(\gamma_{b(x)}\right)} d P_{Y}(y)
$$

The asymptotic efficiency of $\bar{Q}$. In the proof of the next Proposition 4.2 we denote the usual stochastic order by $\leq_{s t}$, and the likelihood ratio order by $\leq_{l r}$; see the book of Müller and Stoyan [MS] (page 2 and page 12) for the definitions.

Proposition 4.2 Assume $(\boldsymbol{R}),(\boldsymbol{M})$ and the hypotheses of Proposition 3.4. Then the law $\bar{Q}$ is admissible for simulation, i.e. $\bar{Q}\left(\tau_{u}^{\alpha}<\infty\right)=1$ for all $u, \alpha>0$.

Proof. We prove the admissibility of $\bar{Q}$ showing that, for all $u, \alpha>0, \lim \sup _{t \rightarrow \infty} R^{\alpha}(t)=-\infty$.

Throughout this proof we simply write $\widetilde{R}(t)$ in place of $\widetilde{R}^{1}(t)$. We recall that the points of $\left(N^{\rho}(t)\right)$ are denoted by $\left(T_{n}\right)$; the points of $\left(N^{\lambda}(t)\right)$ will be denoted by $\left(T_{n}^{\lambda}\right)$. We notice that, under $\bar{Q},\left(N^{\lambda}(t)\right)$ and $\left(N^{\rho}(t)\right)$ are Cox processes with intensities $\left(\left.\lambda M_{X^{\lambda}}\left(\gamma_{b(y)}\right)\right|_{y=\widetilde{R}(t)}\right)$ and $\left(\left.\rho M_{\widehat{X}}\left(\gamma_{b(y)}\right)\right|_{y=\widetilde{R}(t)}\right)$ respectively. Then, by a standard procedure (see e.g. Müller and Stoyan [MS], pp. 211-216), on the same probability space we can define two independent homogeneous Poisson processes $\left(N^{\lambda_{*}}(t)\right)$ and $\left(N^{\rho_{*}}(t)\right)$ with intensities $\lambda_{*}=\lambda M_{X^{\lambda}}\left(\gamma_{\underline{b}}\right)$ and $\rho_{*}=\rho M_{\widehat{X}}\left(\gamma_{\underline{b}}\right)$ respectively; moreover the points of $\left(N^{\lambda_{*}}(t)\right)$ are a subset of the points of $\left(N^{\lambda}(t)\right)$ and the points of $\left(N^{\rho_{*}}(t)\right)$ are a subset of the points of $\left(N^{\rho}(t)\right)$. Therefore we have

$$
S(t)=\sum_{n=1}^{N^{\lambda}(t)} X_{n}^{\lambda}+\sum_{n=1}^{N^{\rho}(t)} \sum_{i=1}^{N^{(n)}(t)} X_{i}^{(n)} \geq \sum_{n=1}^{N^{\lambda_{*}}(t)} X_{n}^{\lambda}+\sum_{n=1}^{N^{\rho_{*}(t)}} \sum_{i=1}^{N^{(n)}(t)} X_{i}^{(n)}, \bar{Q} \text { a.s.. }
$$

On the same probability space we can also define two independent sequences of i.i.d. random variables $\left(V_{n}^{\lambda}\right)$ and $\left(V_{n}^{\rho}\right)$, independent of $\left(N^{\lambda_{*}}(t)\right)$ and $\left(N^{\rho_{*}}(t)\right)$, such that the (common) law $\bar{Q}_{V^{\lambda}}$ of $\left(V_{n}^{\lambda}\right)$ and the (common) law $\bar{Q}_{V^{\rho}}$ of $\left(V_{n}^{\rho}\right)$ are defined by

$$
d \bar{Q}_{V^{\lambda}}(z)=\frac{e^{\gamma_{\underline{b}} z}}{M_{X^{\lambda}}\left(\gamma_{\underline{b}}\right)} d P_{X^{\lambda}}(z) \text { and } d \bar{Q}_{V^{\rho}}(z)=\frac{e^{\gamma_{\underline{b}} z}}{M_{\widehat{X}}\left(\gamma_{\underline{b}}\right)} d P_{\widehat{X}}(z)
$$

$\left(P_{X^{\lambda}}\right.$ is the common law of the random variables $\left(X_{n}^{\lambda}\right)$ and $P_{\widehat{X}}$ is the common law of the random variables $\left(\widehat{X}_{n}\right)$, under the original probability measure $\left.P\right)$. Thus, we have $V_{n}^{\lambda} \leq_{l r}\left[X_{n}^{\lambda} \mid\left\{\widetilde{R}\left(T_{n}^{\lambda}\right)=x\right\}\right]$ and therefore $V_{n}^{\lambda} \leq_{s t} X_{n}^{\lambda}$ (see e.g. Müller and Stoyan [MS], Theorems 1.4.5 and 1.3.8); similarly we have $V_{n}^{\rho} \leq_{l r}\left[\widehat{X}_{n} \mid\left\{\widetilde{R}\left(T_{n}\right)=x\right\}\right]$ and therefore $V_{n}^{\rho} \leq_{s t} \widehat{X}_{n}$. Thus, by a well-known result on the usual stochastic order (see e.g. Müller and Stoyan [MS], Theorem 1.2.4), we can think that

$$
V_{n}^{\lambda} \leq X_{n}^{\lambda} \text { and } V_{n}^{\rho} \leq \widehat{X}_{n}, \bar{Q} \text { a.s.. }
$$

Then we have

$$
\begin{gathered}
\liminf _{t \rightarrow \infty} \frac{S(t)}{t} \geq{ }^{\text {by }(15)} \liminf _{t \rightarrow \infty} \frac{\sum_{n=1}^{N^{\lambda_{*}}(t)} X_{n}^{\lambda}+\sum_{n=1}^{N^{\rho_{*}}(t)} \sum_{i=1}^{N^{(n)}(t)} X_{i}^{(n)}}{t}= \\
=\liminf _{t \rightarrow \infty} \frac{\sum_{n=1}^{N^{\lambda *}(t)} X_{n}^{\lambda}+\sum_{n=1}^{N^{\rho *}(t)} \widehat{X}_{n}-\sum_{n=1}^{N^{\rho *}(t)}\left(\widehat{X}_{n}-\sum_{i=1}^{N^{(n)}(t)} X_{i}^{(n)}\right)}{t} \geq{ }^{\text {by (16) }} \\
\geq \liminf _{t \rightarrow \infty} \frac{\sum_{n=1}^{N^{\lambda *}(t)} V_{n}^{\lambda}+\sum_{n=1}^{N^{\rho *}(t)} V_{n}^{\rho}-\sum_{n=1}^{N^{\rho *}(t)}\left(\sum_{i=N^{(n)}(t)+1}^{N^{(n)}(\infty)} X_{i}^{(n)}\right)}{t} \geq
\end{gathered}
$$




$$
\geq \lambda_{*} \mathbb{E}_{\bar{Q}}\left[V_{1}^{\lambda}\right]+\rho_{*} \mathbb{E}_{\bar{Q}}\left[V_{1}^{\rho}\right]=\lambda M_{X^{\lambda}}\left(\gamma_{\underline{b}}\right) \frac{M_{X^{\lambda}}^{\prime}\left(\gamma_{\underline{b}}\right)}{M_{X^{\lambda}}\left(\gamma_{\underline{b}}\right)}+\rho M_{\widehat{X}}\left(\gamma_{\underline{b}}\right) \frac{M_{\widehat{X}}^{\prime}\left(\gamma_{\underline{b}}\right)}{M_{\widehat{X}}\left(\gamma_{\underline{b}}\right)}=\Lambda^{\prime}\left(\gamma_{\underline{b}}\right) \bar{Q} \text { a.s.; }
$$

we remark that the key inequality $(\star)$ holds since, for each $n \geq 1$, we have $\sum_{i=N^{(n)}(t)+1}^{N^{(n)}(\infty)} X_{i}^{(n)}=0$ for $t>t_{\max }$ by $(\mathbf{R})$.

Finally, we notice that, $\bar{Q}$ almost surely, we have

$$
\limsup _{t \rightarrow \infty} \frac{R^{\alpha}(t)}{t} \leq \limsup _{t \rightarrow \infty} \frac{1}{t} \int_{0}^{t} b\left(R^{\alpha}(s)\right) d s-\liminf _{t \rightarrow \infty} \frac{S(\alpha t)}{\alpha t} \leq B-\Lambda^{\prime}\left(\gamma_{\underline{b}}\right)<0,
$$

where the latter inequality is guaranteed by $(\mathbf{M})$.

Proposition 4.3 Assume the hypotheses of Proposition 4.2. Then $\bar{Q}$ is an asymptotically efficient simulation law, i.e. $\bar{Q}$ is admissible and (14) holds with $Q=\bar{Q}$ for all $u>0$.

Proof. The admissibility of $\bar{Q}$ has been proved in the previous Proposition 4.2. We have to check (14) with $Q=\bar{Q}$.

Lower bound. Using Jensen's inequality and Proposition 4.1 we have

$$
\begin{gathered}
\liminf _{\alpha \rightarrow \infty} \frac{1}{\alpha} \log \eta_{\bar{Q}}(\alpha)=\liminf _{\alpha \rightarrow \infty} \frac{1}{\alpha} \log \mathbb{E}_{\bar{Q}}\left[\left(\frac{d P_{\tau_{u}^{\alpha}}^{\alpha}}{d \bar{Q}_{\tau_{u}^{\alpha}}^{\alpha}}\right)^{2} 1_{\tau_{u}^{\alpha}<\infty}\right] \geq \\
\geq 2 \liminf _{\alpha \rightarrow \infty} \frac{1}{\alpha} \log \mathbb{E}_{\bar{Q}}\left[\frac{d P_{\tau_{u}^{\alpha}}^{\alpha}}{d \bar{Q}_{\tau_{u}^{\alpha}}^{\alpha}} 1_{\tau_{u}^{\alpha}<\infty}\right]=2 \liminf _{\alpha \rightarrow \infty} \frac{1}{\alpha} \log \Psi_{\alpha}(u)=-2 \int_{0}^{u} \gamma_{b(x)} d x .
\end{gathered}
$$

Upper bound. The following preliminary inequality holds:

$$
\int_{0}^{\tau_{u}^{\alpha}} \gamma_{b\left(\widetilde{R}^{\alpha}(s-)\right)} d \widetilde{R}^{\alpha}(s) \leq-f(u), \text { with } f(u)=\int_{0}^{u} \gamma_{b(x)} d x
$$

The proof of (17) is similar to the proof of formula (33) of Ganesh, Macci and Torrisi [GMT]: we have to consider a suitable integration by parts formula, the monotonicity of $b(\cdot)$ (see condition (M)) and the monotonicity of $b \mapsto \gamma_{b}$. Thus

$$
\begin{gathered}
\limsup _{\alpha \rightarrow \infty} \frac{1}{\alpha} \log \eta_{\bar{Q}}(\alpha)=\limsup _{\alpha \rightarrow \infty} \frac{1}{\alpha} \log \mathbb{E}_{\bar{Q}}\left[\exp \left(2 \alpha \int_{0}^{\tau_{u}^{\alpha}} \gamma_{b\left(\widetilde{R}^{\alpha}(s-)\right)} d \widetilde{R}^{\alpha}(s)\right) 1_{\tau_{u}^{\alpha}<\infty}\right] \leq{ }^{\text {by }(17)} \\
\leq \limsup _{\alpha \rightarrow \infty} \frac{1}{\alpha} \log \mathbb{E}_{\bar{Q}}[\exp (-2 \alpha f(u))]=-2 f(u)=-2 \int_{0}^{u} \gamma_{b(x)} d x
\end{gathered}
$$

Remark 4.4 We remark that, for each fixed $\alpha, u>0$, we have $\Psi_{\alpha}(u) \leq \exp \left(-\alpha \int_{0}^{u} \gamma_{b(x)} d x\right)$. This easily follows by $\Psi_{\alpha}(u)=\mathbb{E}_{\bar{Q}}\left[\exp \left(\alpha \int_{0}^{\tau_{u}^{\alpha}} \gamma_{b\left(\widetilde{R}^{\alpha}(s-)\right)} d \widetilde{R}^{\alpha}(s)\right) 1_{\tau_{u}^{\alpha}<\infty}\right]$ and (17), and corresponds to the Lundberg inequality in our context.

\section{$5 \quad$ Bayesian estimates for ruin probabilities}

Throughout this section we assume that, as in Albrecher and Asmussen [AA], the distribution of the random variables $\left(X_{n}^{\lambda}\right)$ coincides with the distribution of the random variables $\left(X_{i}^{(n)}\right)$. Thus, we write

$$
S(t)=\sum_{n=1}^{N(t)} X_{n}
$$


where $\left(X_{n}\right)$ are i.i.d. random variables, independent of $(N(t))$; moreover

$$
\Lambda(\gamma)=\lambda\left(M_{X}(\gamma)-1\right)+\rho\left(M_{\widehat{X}}(\gamma)-1\right)
$$

where $M_{X}(\gamma)=\mathbb{E}\left[e^{\gamma X_{n}}\right]$ is the common moment generating function of the random variables $\left(X_{n}\right)$.

Furthermore we always think that the unknown parameter is the (common) law of the claims $\ell$, and we consider the following restriction:

$(\mathbf{V})$ : we have $\ell([0, V])=1$ for some known constant $V \in(0, \infty)$.

In other words, for some known $V \in(0, \infty)$, we require that $\ell \in M_{1}[0, V]$ where $M_{1}[0, V]$ is the family of all probability measures on $[0, V]$. We think $M_{1}[0, V]$ equipped with the topology of weak convergence.

As a prior distribution on $\ell$ we choose a Dirichlet process prior $\pi$; it is known that the posterior distribution $\pi_{n}$ after the first $n$ sampled claims $X_{1}, \ldots, X_{n}$ is again a Dirichlet process (see Ferguson $[F]$ ). An essential ingredient in the proof of Proposition 5.2 presented below is the LDP for posterior Dirichlet processes on a compact space proved by Ganesh and O'Connell [GO2]; for this reason we need to consider assumption (V).

In what follows we use the symbols $\Psi_{\alpha}^{(\ell)}(u), \Lambda_{\ell}, \mathcal{G}_{\ell}$ and $\gamma_{b}^{(\ell)}$ in place of $\Psi_{\alpha}(u), \Lambda, M_{X}$ and $\gamma_{b}$ respectively; thus, in particular, the predictive ruin probability is

$$
\int_{M_{1}[0, V]} \Psi_{\alpha}^{(\ell)}(u) \pi_{n}(d \ell)
$$

We remark that we defined the value $\gamma_{b}^{(\ell)}$ when $(\mathbf{S})$ and $(\mathbf{N})$ hold (see condition $(\mathbf{C})$ ); here it is useful to consider the more general definition

$$
\gamma_{b}^{(\ell)}=\sup \left\{\gamma \geq 0: \Lambda_{\ell}(\gamma)-b \gamma \leq 0\right\}
$$

indeed in such a case we have $\gamma_{b}^{(\ell)} \geq 0, \Lambda_{\ell}\left(\gamma_{b}^{(\ell)}\right)-b \gamma_{b}^{(\ell)}=0$ and $\gamma_{b}^{(\ell)}>0$ if and only if $b>\Lambda_{\ell}^{\prime}(0)$.

In view of what follows it is useful to consider the function $\Lambda_{\ell, \rho=0}$ and the value $\gamma_{b, \rho=0}^{(\ell)}$, i.e. $\Lambda_{\ell}$ and $\gamma_{b}^{(\ell)}$ when $\rho=0$ :

$$
\Lambda_{\ell, \rho=0}(\gamma)=\lambda\left(\mathcal{G}_{\ell}(\gamma)-1\right) ; \gamma_{b, \rho=0}^{(\ell)}=\sup \left\{\gamma \geq 0: \Lambda_{\ell, \rho=0}(\gamma)-b \gamma \leq 0\right\}
$$

In particular we have $\gamma_{b, \rho=0}^{(\ell)} \geq \gamma_{b}^{(\ell)}$.

The aim of this section is to provide two asymptotic bounds for

$$
\int_{M_{1}[0, V]} \Psi_{n q}^{(\ell)}(u) \pi_{n}(d \ell)
$$

as we shall see the upper bound is sharper than the lower bound. We start with the following lemma.

Lemma 5.1 Let $b:[0, u] \rightarrow\left[b_{-}, b_{+}\right]$be a measurable function, where $b_{+}>b_{-}>0$. Then the function $\ell \in M_{1}[0, V] \mapsto \int_{0}^{u} \gamma_{b(x)}^{(\ell)} d x$ is continuous.

Proof. As a first step we show the uniform continuity of the following function:

$$
(\ell, b) \in M_{1}[0, V] \times\left[b_{-}, b_{+}\right] \mapsto \gamma_{b}^{(\ell)} .
$$

We notice that $M_{1}[0, V]$ is a compact set (see e.g. Dembo and Zeitouni [DZ], Theorem D.8, pages 355-356) and therefore the domain of the function (18) is a compact set (since it is the product between two compact sets); thus we only need to check the continuity of the function (18). 
Let $\widetilde{\rho}$ be the Prohorov's metric. We want to show that $\lim _{n \rightarrow \infty} \gamma_{b_{n}}^{\left(\ell_{n}\right)}=\gamma_{b_{\infty}}^{\left(\ell_{\infty}\right)}$, when $\lim _{n \rightarrow \infty} \widetilde{\rho}\left(\ell_{n}, \ell_{\infty}\right)=$ 0 and $\lim _{n \rightarrow \infty} b_{n}=b_{\infty}$ for some $\ell_{\infty} \in M_{1}[0, V]$ and $b_{\infty} \in\left[b_{-}, b_{+}\right]$. First of all, since $[0, V]$ is compact, we have $\lim _{n \rightarrow \infty} \mathcal{G}_{\ell_{n}}(\gamma)=\mathcal{G}_{\ell_{\infty}}(\gamma)$ for all $\gamma \in \mathbb{R}$; thus

$$
\lim _{n \rightarrow \infty} \Lambda_{\ell_{n}}(\gamma)-b_{n} \gamma=\Lambda_{\ell_{\infty}}(\gamma)-b_{\infty} \gamma \text { for all } \gamma \in \mathbb{R}
$$

Moreover $\Lambda_{\ell_{n}}\left(\gamma_{b_{n}}^{\left(\ell_{n}\right)}\right)-b_{n} \gamma_{b_{n}}^{\left(\ell_{n}\right)}=0$ for all $n \geq 1$ and $\Lambda_{\ell_{\infty}}\left(\gamma_{b_{\infty}}^{\left(\ell_{\infty}\right)}\right)-b_{\infty} \gamma_{b_{\infty}}^{\left(\ell_{\infty}\right)}=0$. We notice that there exist $\beta_{-}, \beta_{+} \in \mathbb{R}$ (which depends on $b_{\infty}$ and $\ell_{\infty}$ ) such that $\beta_{-}<\gamma_{b_{\infty}}^{\left(\ell_{\infty}\right)}<\beta_{+}$and $\Lambda_{\ell_{\infty}}\left(\beta_{-}\right)-b_{\infty} \beta_{-}<$ 0 and $\Lambda_{\ell_{\infty}}\left(\beta_{+}\right)-b_{\infty} \beta_{+}>0$; as a consequence there exists $\bar{n} \geq 1$ such that $\beta_{-}<\gamma_{b_{n}}^{\left(\ell_{n}\right)}<\beta_{+}$ for all $n \geq \bar{n}$. Thus, there exists a subsequence $\left(\gamma_{b_{n(k)}}^{\left(\ell_{n(k)}\right)}\right)$ such that $\lim _{k \rightarrow \infty} \gamma_{b_{n(k)}}^{\left(\ell_{n(k)}\right)}=\bar{\gamma}$ for some $\bar{\gamma} \in\left[\beta_{-}, \beta_{+}\right]$. Our aim is to show that $\bar{\gamma}=\gamma_{b_{\infty}}^{\left(\ell_{\infty}\right)}$. For this we reason by contradiction, distinguishing two cases.

1) If $\bar{\gamma}<\gamma_{b_{\infty}}^{\left(\ell_{\infty}\right)}$ there exists $\gamma_{-} \in\left(\bar{\gamma}, \gamma_{b_{\infty}}^{\left(\ell_{\infty}\right)}\right)$ such that $\gamma_{b_{n(k)}}^{\left(\ell_{n(k)}\right)}<\gamma_{-}$eventually, and we have the following contradiction:

$$
\begin{aligned}
& 0=\Lambda_{\ell_{n(k)}}\left(\gamma_{b_{n(k)}}^{\left(\ell_{n(k)}\right)}\right)-b_{n(k)} \gamma_{b_{n(k)}}^{\left(\ell_{n(k)}\right)}<\Lambda_{\ell_{n(k)}}\left(\gamma_{-}\right)-b_{n(k)} \gamma_{-}, \\
& \text {and } \lim _{k \rightarrow \infty} \Lambda_{\ell_{n(k)}}\left(\gamma_{-}\right)-b_{n(k)} \gamma_{-}=\Lambda_{\ell_{\infty}}\left(\gamma_{-}\right)-b_{\infty} \gamma_{-}<\Lambda_{\ell_{\infty}}\left(\gamma_{b_{\infty}}^{\left(\ell_{\infty}\right)}\right)-b_{\infty} \gamma_{b_{\infty}}^{\left(\ell_{\infty}\right)}=0 ;
\end{aligned}
$$

2) If $\bar{\gamma}>\gamma_{b_{\infty}}^{\left(\ell_{\infty}\right)}$ there exists $\gamma_{+} \in\left(\gamma_{b_{\infty}}^{\left(\ell_{\infty}\right)}, \bar{\gamma}\right)$ such that $\gamma_{b_{n(k)}}^{\left(\ell_{n(k)}\right)}>\gamma_{+}$eventually, and we have the following contradiction:

$$
\begin{aligned}
& 0=\Lambda_{\ell_{n(k)}}\left(\gamma_{b_{n(k)}}^{\left(\ell_{n(k)}\right)}\right)-b_{n(k)} \gamma_{b_{n(k)}}^{\left(\ell_{n(k)}\right)}>\Lambda_{\ell_{n(k)}}\left(\gamma_{+}\right)-b_{n(k)} \gamma_{+}, \\
& \text {and } \lim _{k \rightarrow \infty} \Lambda_{\ell_{n(k)}}\left(\gamma_{+}\right)-b_{n(k)} \gamma_{+}=\Lambda_{\ell_{\infty}}\left(\gamma_{+}\right)-b_{\infty} \gamma_{+}>\Lambda_{\ell_{\infty}}\left(\gamma_{b_{\infty}}^{\left(\ell_{\infty}\right)}\right)-b_{\infty} \gamma_{b_{\infty}}^{\left(\ell_{\infty}\right)}=0 .
\end{aligned}
$$

We finally show that any convergent subsequence of $\left(\gamma_{b_{n}}^{\left(\ell_{n}\right)}\right)$ converges to $\gamma_{b_{\infty}}^{\left(\ell_{\infty}\right)}$. We reason by contradiction. Suppose that there exists $\varepsilon_{0}>0$ and a subsequence $\left(\gamma_{b_{n(k)}}^{\left(\ell_{n(k)}\right)}\right)$ of $\left(\gamma_{b_{n}}^{\left(\ell_{n}\right)}\right)$ such that $\left|\gamma_{b_{n(k)}}^{\left(\ell_{n(k)}\right)}-\gamma_{b_{\infty}}^{\left(\ell_{\infty}\right)}\right| \geq \varepsilon_{0}$ eventually. Then, since $\left(\gamma_{b_{n(k)}}^{\left(\ell_{n(k)}\right)}\right) \subset\left[\beta_{-}, \beta_{+}\right]$, there exists a subsequence of $\left(\gamma_{b_{n(k)}}^{\left(\ell_{n(k)}\right)}\right)$ which converges to $\gamma_{b_{\infty}}^{\left(\ell_{\infty}\right)}$ by 1$)$ and 2$)$. This yields a contradiction.

The uniform continuity of the function (18) is proved. Then for all $\varepsilon>0$ there exists $\delta_{\varepsilon}>0$ such that, if $\widetilde{\rho}\left(\ell, \ell_{\infty}\right)<\delta_{\varepsilon}$, we have

$$
\sup _{b \in\left[b_{-}, b_{+}\right]}\left|\gamma_{b}^{(\ell)}-\gamma_{b}^{\left(\ell_{\infty}\right)}\right| \leq \frac{\varepsilon}{u} .
$$

Let $\left(\ell_{n}\right) \subset M_{1}[0, V]$ be such that $\lim _{n \rightarrow \infty} \widetilde{\rho}\left(\ell_{n}, \ell_{\infty}\right)=0$ for some $\ell_{\infty} \in M_{1}[0, V]$. We have to check that $\lim _{n \rightarrow \infty} \int_{0}^{u} \gamma_{b(x)}^{\left(\ell_{n}\right)} d x=\int_{0}^{u} \gamma_{b(x)}^{\left(\ell_{\infty}\right)} d x$. For all $\varepsilon>0$ there exists $\bar{n} \geq 1$ such that $\widetilde{\rho}\left(\ell_{n}, \ell_{\infty}\right)<$ $\delta_{\varepsilon}$ for all $n \geq \bar{n}$; thus, by (19),

$$
\left|\int_{0}^{u} \gamma_{b(x)}^{\left(\ell_{n}\right)} d x-\int_{0}^{u} \gamma_{b(x)}^{\left(\ell_{\infty}\right)} d x\right| \leq \int_{0}^{u}\left|\gamma_{b(x)}^{\left(\ell_{n}\right)}-\gamma_{b(x)}^{\left(\ell_{\infty}\right)}\right| d x \leq u \sup _{b \in\left[b_{-}, b_{+}\right]}\left|\gamma_{b}^{\left(\ell_{n}\right)}-\gamma_{b}^{\left(\ell_{\infty}\right)}\right| \leq \varepsilon .
$$

In view of the asymptotic bounds for the predictive ruin probability presented below, it is useful to consider the relative entropy of $\widehat{\ell}$ with respect to $\ell$ defined by

$$
H(\widehat{\ell} \mid \ell)= \begin{cases}\int_{[0, V]} \frac{d \widehat{\ell}}{d \ell}(x) \widehat{\ell}(d x) & \text { if } \widehat{\ell} \ll \ell \text { with density } \frac{d \widehat{\ell}}{d \ell} \\ \infty & \text { otherwise }\end{cases}
$$


Indeed, the lower bound and the upper bound will be expressed in terms of the functions

$$
a_{+}(q, u \mid \widehat{\ell}):=\sup _{\ell \in M_{1}[0, V]}\left[-q \int_{0}^{u} \gamma_{b(x)}^{(\ell)} d x-H(\widehat{\ell} \mid \ell)\right] \text { and } a_{-}(q, u \mid \widehat{\ell}):=\sup _{\ell \in M_{1}[0, V]}\left[-q u \gamma_{B, \rho=0}^{(\ell)}-H(\widehat{\ell} \mid \ell)\right]
$$

where $q, u>0$ and $\left.\widehat{\ell} \in M_{1}[0, V]\right)$.

Proposition 5.2 Assume the hypotheses of Proposition 4.2 and (V). Moreover, assume that the empirical law $\frac{1}{n} \sum_{i=1}^{n} \delta_{X_{i}}$ (of the first $n$ sampled claims $X_{1}, \ldots, X_{n}$ ) converges to $\widehat{\ell} \in M_{1}[0, V]$ as $n \rightarrow \infty$. Then, for all $q, u>0$, we have:

$$
\begin{aligned}
(\mathbf{L B}): & \liminf _{n \rightarrow \infty} \frac{1}{n} \log \int_{M_{1}[0, V]} \Psi_{n q}^{(\ell)}(u) \pi_{n}(d \ell) \geq a_{-}(q, u \mid \widehat{\ell}) \\
(\mathbf{U B}): & \limsup _{n \rightarrow \infty} \frac{1}{n} \log \int_{M_{1}[0, V]} \Psi_{n q}^{(\ell)}(u) \pi_{n}(d \ell) \leq a_{+}(q, u \mid \widehat{\ell}) .
\end{aligned}
$$

Proof. The idea is to prove (LB) and (UB) applying Varadhan's Lemma (see e.g. Dembo and Zeitouni [DZ], Theorem 4.3.1, page 137). Indeed, it is known (see Ganesh and O'Connell [GO2], Theorem 1) that, under our hypotheses, $\left(\pi_{n}\right)$ satisfies the LDP with rate function $H(\widehat{\ell} \mid \cdot)$.

Proof of $(\mathbf{L B})$. Let $\left(S_{\rho=0}(t)\right)$ be the process defined by $S_{\rho=0}(t)=\sum_{n=1}^{N^{\lambda}(t)} X_{n}^{\lambda}$ (we are referring to equation (3)) and let $\Psi_{B, \rho=0}^{(\ell)}(u)$ be the ruin probability defined by

$$
\Psi_{B, \rho=0}^{(\ell)}(u)=P\left(\exists t>0: u+B t-S_{\rho=0}(t) \leq 0\right)
$$

since $P\left(X_{n}^{\lambda} \leq V\right)=1$ for all $n \geq 1$ by $(\mathbf{V})$, we have $\Psi_{B, \rho=0}^{(\ell)}(u) \geq e^{-(u+V) \gamma_{B, \rho=0}^{(\ell)}}$. Thus, since $b(x) \leq B$ by $(\mathbf{M})$, we get

$$
\begin{gathered}
\Psi_{\alpha}^{(\ell)}(u) \geq P\left(\exists t>0: u+B t-\frac{S_{\rho=0}(\alpha t)}{\alpha} \leq 0\right)= \\
=P\left(\exists t>0: \alpha u+B \alpha t-S_{\rho=0}(\alpha t) \leq 0\right)=\Psi_{B, \rho=0}^{(\ell)}(\alpha u) \geq e^{-(\alpha u+V) \gamma_{B, \rho=0}^{(\ell)}} .
\end{gathered}
$$

In particular $\Psi_{n q}^{(\ell)}(u) \geq e^{-(n q u+V) \gamma_{B, \rho=0}^{(\ell)}}=e^{-n\left(q u+\frac{V}{n}\right) \gamma_{B, \rho=0}^{(\ell)}}$; therefore, for all $\varepsilon>0$, there exists $\bar{n} \geq 1$ such that

$$
\Psi_{n q}^{(\ell)}(u) \geq e^{-n(q u+\varepsilon) \gamma_{B, \rho=0}^{(\ell)}} \text { for all } n \geq \bar{n}
$$

whence we obtain

$$
\liminf _{n \rightarrow \infty} \frac{1}{n} \log \int_{M_{1}[0, V]} \Psi_{n q}^{(\ell)}(u) \pi_{n}(d \ell) \geq \liminf _{n \rightarrow \infty} \frac{1}{n} \log \int_{M_{1}[0, V]} e^{-n(q u+\varepsilon) \gamma_{B, \rho=0}^{(\ell)}} \pi_{n}(d \ell) .
$$

Since $\ell \in M_{1}[0, V] \mapsto \gamma_{B, \rho=0}^{(\ell)}$ is continuous by Lemma 5.1 , and

$$
\limsup _{n \rightarrow \infty} \frac{1}{n} \log \int_{M_{1}[0, V]} e^{-z n(q u+\varepsilon) \gamma_{B, \rho=0}^{(\ell)}} \pi_{n}(d \ell) \leq 0 \text { for all } z>1,
$$

we have

$$
\lim _{n \rightarrow \infty} \frac{1}{n} \log \int_{M_{1}[0, V]} e^{-n(q u+\varepsilon) \gamma_{B, \rho=0}^{(\ell)} \pi_{n}(d \ell)}=\sup _{\ell \in M_{1}[0, V]}\left[-(q u+\varepsilon) \gamma_{B, \rho=0}^{(\ell)}-H(\widehat{\ell} \mid \ell)\right]
$$


by Varadhan's Lemma. Then, by (20) and (21), for all $\varepsilon>0$ we have

$$
\liminf _{n \rightarrow \infty} \frac{1}{n} \log \int_{M_{1}[0, V]} \Psi_{n q}^{(\ell)}(u) \pi_{n}(d \ell) \geq \sup _{\ell \in M_{1}[0, V]}\left[-(q u+\varepsilon) \gamma_{B, \rho=0}^{(\ell)}-H(\widehat{\ell} \mid \ell)\right] .
$$

Finally, we notice that the function

$$
z \in[0, \infty) \mapsto \sup _{\ell \in M_{1}[0, V]}\left[-z \gamma_{B, \rho=0}^{(\ell)}-H(\widehat{\ell} \mid \ell)\right]
$$

is continuous (it is a convex function on $(0, \infty)$ which assumes finite values; moreover it is continuous at $z=0)$, therefore we obtain $(\mathbf{L B})$ by letting $\varepsilon$ go to zero in $(22)$.

Proof of (UB). By Remark 4.4 we have

$$
\int_{M_{1}[0, V]} \Psi_{n q}^{(\ell)}(u) \pi_{n}(d \ell) \leq \int_{M_{1}[0, V]} \exp \left(-n q \int_{0}^{u} \gamma_{b(x)}^{(\ell)} d x\right) \pi_{n}(d \ell) .
$$

Moreover, the function $\ell \in M_{1}[0, V] \mapsto \int_{0}^{u} \gamma_{b(x)}^{(\ell)} d x$ is continuous by Lemma 5.1 and

$$
\limsup _{n \rightarrow \infty} \frac{1}{n} \log \int_{M_{1}[0, V]} \exp \left(-z n q \int_{0}^{u} \gamma_{b(x)}^{(\ell)} d x\right) \pi_{n}(d \ell) \leq 0 \text { for all } z>1 .
$$

Then

$$
\lim _{n \rightarrow \infty} \frac{1}{n} \log \int_{M_{1}[0, V]} \exp \left(-n q \int_{0}^{u} \gamma_{b(x)}^{(\ell)} d x\right) \pi_{n}(d \ell)=a_{+}(q, u \mid \widehat{\ell})
$$

by Varadhan's Lemma, and inequality (UB) follows by (23) and (24).

Firstly we remark that, if the function $b$ is constant and $\rho=0$, the bounds $a_{+}(q, u \mid \widehat{\ell})$ and $a_{-}(q, u \mid \widehat{\ell})$ coincide. Thus Proposition 5.2 extends Proposition 4.1 in Macci and Petrella [MP] which refers to the classical risk process. We also remark that

$$
-q u \gamma_{B, \rho=0}^{(\widehat{\ell})} \leq a_{-}(q, u \mid \widehat{\ell}) \text { and }-q \int_{0}^{u} \gamma_{b(x)}^{(\widehat{\ell})} d x \leq a_{+}(q, u \mid \widehat{\ell}),
$$

and the law $\hat{\ell}$ in the statement of Proposition 5.2 is the true law of the claims $\left(X_{n}\right)$. Thus, the Bayesian bounds are asymptotically more conservative than the corresponding non-Bayesian bounds, with a degree which becomes more pronounced as $q$ increases; indeed, one can prove that the differences

$$
a_{-}(q, u \mid \widehat{\ell})-\left(-q u \gamma_{B, \rho=0}^{(\widehat{\ell})}\right) \text { and } a_{+}(q, u \mid \widehat{\ell})-\left(-q \int_{0}^{u} \gamma_{b(x)}^{(\widehat{\ell})} d x\right)
$$

are nondecreasing with respect to $q$. This fact agrees with the discussion of Ganesh et al. [GGOP] on some network and risk management problems.

\section{Appendix A: The measurability requirement concerning the events in (7)}

As pointed out in Remark (b) just after Definition 4.2.10 in Dembo and Zeitouni [DZ], the measurability requirement concerning this definition is satisfied if we deal with separable real-valued stochastic processes. Thus, as pointed out in [LT] (page 45), it is enough to prove that the continuity in probability of $\frac{\widetilde{S}(\alpha t)}{\alpha}-\frac{S(\alpha t)}{\alpha}$ with respect to $t$. It is easy to check that it is enough to consider the case $\alpha=1$. In what follows we prove the right-continuity; the left-continuity can be proved similarly and we omit the details. Thus, for all $\varepsilon>0$, we shall prove that

$$
\lim _{t \downarrow t_{0}} P\left(\left|\widetilde{S}(t)-S(t)-\left(\widetilde{S}\left(t_{0}\right)-S\left(t_{0}\right)\right)\right|>\varepsilon\right)=0 .
$$


Firstly note that

$$
\begin{gathered}
P\left(\left|\widetilde{S}(t)-S(t)-\left(\widetilde{S}\left(t_{0}\right)-S\left(t_{0}\right)\right)\right|>\varepsilon\right) \leq P\left(\left|\widetilde{S}(t)-\widetilde{S}\left(t_{0}\right)\right|+\left|S(t)-S\left(t_{0}\right)\right|>\varepsilon\right) \leq \\
\leq P\left(\left|\widetilde{S}(t)-\widetilde{S}\left(t_{0}\right)\right|>\frac{\varepsilon}{2}\right)+P\left(\left|S(t)-S\left(t_{0}\right)\right|>\frac{\varepsilon}{2}\right) \leq \frac{2}{\varepsilon}\left(\mathbb{E}\left[\left|\widetilde{S}(t)-\widetilde{S}\left(t_{0}\right)\right|\right]+\mathbb{E}\left[\left|S(t)-S\left(t_{0}\right)\right|\right]\right) ;
\end{gathered}
$$

so, since $t>t_{0}$, we have

$$
P\left(\left|\widetilde{S}(t)-S(t)-\left(\widetilde{S}\left(t_{0}\right)-S\left(t_{0}\right)\right)\right|>\varepsilon\right) \leq \frac{2}{\varepsilon}\left(\mathbb{E}\left[\widetilde{S}(t)-\widetilde{S}\left(t_{0}\right)\right]+\mathbb{E}\left[S(t)-S\left(t_{0}\right)\right]\right),
$$

and we only have to check that

$$
\lim _{t \downarrow t_{0}} \mathbb{E}[\widetilde{S}(t)]=\mathbb{E}\left[\widetilde{S}\left(t_{0}\right)\right]
$$

and

$$
\lim _{t \downarrow t_{0}} \mathbb{E}[S(t)]=\mathbb{E}\left[S\left(t_{0}\right)\right] .
$$

We start noting that $\mathbb{E}[\widetilde{S}(t)]=\lambda t \mathbb{E}\left[X_{1}^{\lambda}\right]+\rho t \mathbb{E}\left[\widehat{X}_{1}\right]$ and $\mathbb{E}[S(t)]=\lambda t \mathbb{E}\left[X_{1}^{\lambda}\right]+\mathbb{E}\left[\sum_{n=1}^{N^{\rho}(t)} \sum_{i=1}^{N^{(n)}(t)} X_{i}^{(n)}\right]$; thus (25) can be immediately checked, and (26) is equivalent to

$$
\lim _{t \downarrow t_{0}} \mathbb{E}\left[\sum_{n=1}^{N^{\rho}(t)} \sum_{i=1}^{N^{(n)}(t)} X_{i}^{(n)}\right]=\mathbb{E}\left[\sum_{n=1}^{N^{\rho}\left(t_{0}\right)} \sum_{i=1}^{N^{(n)}\left(t_{0}\right)} X_{i}^{(n)}\right]
$$

Note that

$$
\mathbb{E}\left[\sum_{n=1}^{N^{\rho}(t)} \sum_{i=1}^{N^{(n)}(t)} X_{i}^{(n)}\right]=\sum_{j \geq 0} \sum_{n=1}^{j} \mathbb{E}\left[\sum_{i=1}^{N^{(n)}(t)} X_{i}^{(n)} \mid N^{\rho}(t)=j\right] \frac{(\rho t)^{j}}{j !} e^{-\rho t} .
$$

Moreover, given the event $\left\{N^{\rho}(t)=j\right\}$, the distribution of $\left(T_{1}, \ldots, T_{j}\right)$ coincides with the distribution of $\left(t U_{1: j}, \ldots, t U_{j: j}\right)$, where $\left(U_{1: j}, \ldots, U_{j: j}\right)$ are the order statistics of i.i.d. uniformly distributed random variables $U_{1}, \ldots, U_{j}$ on $[0,1]$. Thus, for all $n \in\{1, \ldots, j\}$, we have

$$
\mathbb{E}\left[\sum_{i=1}^{N^{(n)}(t)} X_{i}^{(n)} \mid N^{\rho}(t)=j\right]=\mathbb{E}\left[\sum_{i=1}^{M^{(n)}(t)} X_{i}^{(n)}\right],
$$

where $M^{(n)}$ is a Cox process with stochastic intensity $h\left(\cdot-t U_{n: j}, Y_{n}\right)$; then, if we set $a=\mathbb{E}\left[X_{1}^{(1)}\right]$, we have

$$
\begin{aligned}
& \mathbb{E}\left[\sum_{i=1}^{N^{(n)}(t)} X_{i}^{(n)} \mid N^{\rho}(t)=j\right]=a \int_{0}^{t} \mathbb{E}\left[h\left(s-t U_{n: j}, Y_{n}\right)\right] d s= \\
= & a \int_{0}^{t} d s \int_{0}^{1} d x \mathbb{E}\left[h\left(s-t x, Y_{n}\right)\right] \frac{j !}{(n-1) !(j-n) !} x^{n-1}(1-x)^{j-n}= \\
= & \frac{a}{t^{j}} \frac{j !}{(n-1) !(j-n) !} \int_{0}^{t} d s \int_{0}^{t} d r \mathbb{E}\left[h\left(s-r, Y_{1}\right)\right] r^{n-1}(t-r)^{j-n} .
\end{aligned}
$$

Substituting the latter equality in (28) we get

$\mathbb{E}\left[\sum_{n=1}^{N^{\rho}(t)} \sum_{i=1}^{N^{(n)}(t)} X_{i}^{(n)}\right]=\sum_{j \geq 0} \underbrace{\sum_{n=1}^{j} \frac{a}{t^{j}} \frac{j !}{(n-1) !(j-n) !}\left(\int_{0}^{t} d s \int_{0}^{t} d r \mathbb{E}\left[h\left(s-r, Y_{1}\right)\right] r^{n-1}(t-r)^{j-n}\right) \frac{(\rho t)^{j}}{j !} e^{-\rho t} .}_{=: b_{j}(t)}$ 
Note that

$$
\begin{gathered}
b_{j}(t) \leq \frac{a}{t}\left(\int_{0}^{t} d s \int_{0}^{t} d r \mathbb{E}\left[h\left(s-r, Y_{1}\right)\right]\right) \frac{(\rho t)^{j}}{j !} e^{-\rho t} \sum_{n=1}^{j} \frac{j !}{(n-1) !(j-n) !}= \\
=\frac{a}{t}\left(\int_{0}^{t} d s \int_{0}^{t} d r \mathbb{E}\left[h\left(s-r, Y_{1}\right)\right]\right) \frac{(\rho t)^{j}}{j !} e^{-\rho t} 2^{j} \sum_{n=1}^{j} n\left(\begin{array}{c}
j \\
n
\end{array}\right)\left(\frac{1}{2}\right)^{j}= \\
=\frac{a}{2 t}\left(\int_{0}^{t} d s \int_{0}^{t} d r \mathbb{E}\left[h\left(s-r, Y_{1}\right)\right]\right) \frac{(2 \rho t)^{j}}{j !} e^{-\rho t} j .
\end{gathered}
$$

Consequently, for $t_{0} \leq t \leq 2 t_{0}$, we have

$$
b_{j}(t) \leq \frac{a}{2 t_{0}}\left(\int_{0}^{2 t_{0}} d s \int_{0}^{2 t_{0}} d r \mathbb{E}\left[h\left(s-r, Y_{1}\right)\right]\right) \frac{\left(4 \rho t_{0}\right)^{j}}{j !} e^{-\rho t_{0}} j .
$$

Since $\sum_{j \geq 0} \frac{\left(4 \rho t_{0}\right)^{j}}{j !} j=4 \rho t_{0} e^{4 \rho t_{0}}<\infty$, the dominated convergence theorem yields

$$
\lim _{t \downarrow t_{0}} \mathbb{E}\left[\sum_{n=1}^{N^{\rho}(t)} \sum_{i=1}^{N^{(n)}(t)} X_{i}^{(n)}\right]=\sum_{j \geq 0} \lim _{t \downarrow t_{0}} b_{j}(t)
$$

and (27) follows by the continuity of the functions $\left\{b_{j}(\cdot): j \geq 0\right\}$.

\section{Appendix B: The proof of (10)}

Let $\theta>0$ be arbitrarily fixed. Since the random variables $\left(A_{m}\right)$ are nonnegative we have

$$
\liminf _{m \rightarrow \infty} \frac{1}{m} \log \mathbb{E}\left[e^{\theta A_{m}}\right] \geq 0 .
$$

We shall show that $\limsup _{m \rightarrow \infty} \frac{1}{m} \log \mathbb{E}\left[e^{\theta A_{m}}\right] \leq 0$. By the dominated convergence theorem we have

$$
\lim _{t \rightarrow \infty} \mathbb{E}\left[e^{\theta\left(\sum_{i=1}^{N_{j}^{*}(\infty)} X_{i}^{(j+1)}-\sum_{i=1}^{N_{j}^{*}(t)} X_{i}^{(j+1)}\right)}\right]=1,
$$

for each fixed $j \geq 1$; note that dominated convergence theorem can be applied since

$$
\mathbb{E}\left[e^{\theta\left(\sum_{i=1}^{N_{j}^{*}(\infty)} X_{i}^{(j+1)}-\sum_{i=1}^{N_{j}^{*}(t)} X_{i}^{(j+1)}\right)}\right] \leq \mathbb{E}\left[e^{\theta \sum_{i=1}^{N_{j}^{*}(\infty)} X_{i}^{(j+1)}}\right]=M_{H(\infty, Y)}\left(M_{X}(\theta)-1\right)<\infty
$$

by (S). Thus, for any $\varepsilon>0$ arbitrarily fixed, there exists $t_{\varepsilon}$ such that

$$
\mathbb{E}\left[e^{\theta\left(\sum_{i=1}^{N_{j}^{*}(\infty)} X_{i}^{(j+1)}-\sum_{i=1}^{N_{j}^{*}(t)} X_{i}^{(j+1)}\right)}\right] \leq e^{\varepsilon} \text { for all } t \geq t_{\varepsilon} .
$$

We now show that

$$
\lim _{m \rightarrow \infty} \frac{1}{m} \log P\left(T_{[m \varepsilon]} \leq t_{\varepsilon}\right)=-\infty .
$$

Let $\eta>0$ be arbitrarily fixed. Then

$$
P\left(N^{\rho}\left(t_{\varepsilon}\right) \geq[m \varepsilon]\right) \leq e^{-\eta[m \varepsilon]} \mathbb{E}\left[e^{\eta N^{\rho}\left(t_{\varepsilon}\right)}\right]=e^{-\eta[m \varepsilon]+\rho t_{\varepsilon}\left(e^{\eta}-1\right)},
$$

and therefore $\left.\lim \sup _{m \rightarrow \infty} \frac{1}{m} \log P\left(N^{\rho}\left(t_{\varepsilon}\right)\right) \geq[m \varepsilon]\right) \leq-\eta \varepsilon$; thus, since $\eta>0$ is arbitrary,

$$
\lim _{m \rightarrow \infty} \frac{1}{m} \log P\left(N^{\rho}\left(t_{\varepsilon}\right) \geq[m \varepsilon]\right)=-\infty
$$


and this inequality is equivalent to (30) since we have $P\left(T_{[m \varepsilon]} \leq t_{\varepsilon}\right)=P\left(N^{\rho}\left(t_{\varepsilon}\right) \geq[m \varepsilon]\right)$.

Now, consider the equality

$$
\mathbb{E}\left[e^{\theta A_{m}}\right]=\mathbb{E}\left[e^{\theta A_{m}} 1_{T_{[m \varepsilon]}<t_{\varepsilon}}\right]+\mathbb{E}\left[e^{\theta A_{m}} 1_{T_{[m \varepsilon]} \geq t_{\varepsilon}}\right] .
$$

For the first addendum in (31) we have

$$
\mathbb{E}\left[e^{\theta A_{m}} 1_{T_{[m \varepsilon]}<t_{\varepsilon}}\right] \leq \mathbb{E}\left[e^{\theta \sum_{j=0}^{m-1} \sum_{i=1}^{N_{j}^{*}(\infty)} X_{i}^{(j+1)}} 1_{T_{[m \varepsilon]}<t_{\varepsilon}}\right]=\left(M_{H(\infty, Y)}\left(M_{X}(\theta)-1\right)\right)^{m} P\left(T_{[m \varepsilon]}<t_{\varepsilon}\right),
$$

since $\theta>0, \sum_{j=0}^{m-1} \sum_{i=1}^{N_{j}^{*}(\infty)} X_{i}^{(j+1)}$ and $T_{[m \varepsilon]}$ are independent, and $\left(\sum_{i=1}^{N_{j}^{*}(\infty)} X_{i}^{(j+1)}\right)_{j=0, \ldots, m-1}$ are i.i.d. random variables with common moment generating function $M_{H(\infty, Y)}\left(M_{X}(\cdot)-1\right)$; thus

$$
\lim _{m \rightarrow \infty} \frac{1}{m} \log \mathbb{E}\left[e^{\theta A_{m}} 1_{T_{[m \varepsilon]}<t_{\varepsilon}}\right]=-\infty
$$

by (S) and (30). For the second addendum in (31) we have

$$
\begin{aligned}
& \mathbb{E}\left[e^{\theta A_{m}} 1_{T_{[m \varepsilon]} \geq t_{\varepsilon}}\right] \leq \mathbb{E}\left[e^{\theta\left\{\sum_{j=0}^{[m \varepsilon]-1} \sum_{i=1}^{N_{j}^{*}(\infty)} X_{i}^{(j+1)}+\sum_{j=[m \varepsilon]}^{m-1}\left[\sum_{i=1}^{N_{j}^{*}(\infty)} X_{i}^{(j+1)}-\sum_{i=1}^{N_{j}^{*}\left(t_{\varepsilon}\right)} X_{i}^{(j+1)}\right]\right\}} 1_{T_{[m \varepsilon]} \geq t_{\varepsilon}}\right]=
\end{aligned}
$$

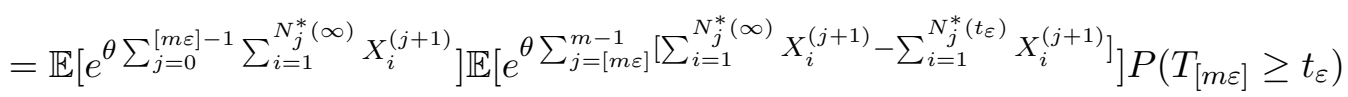

since $\theta>0$, and by the independence of the involved random variables; thus by (29)

$$
\mathbb{E}\left[e^{\theta A_{m}} 1_{T_{[m \varepsilon]} \geq t_{\varepsilon}}\right] \leq\left(M_{H(\infty, Y)}\left(M_{X}(\theta)-1\right)\right)^{[m \varepsilon]}\left(e^{\varepsilon}\right)^{m-[m \varepsilon]}
$$

since $\left(\sum_{i=1}^{N_{j}^{*}(\infty)} X_{i}^{(j+1)}\right)_{j=0, \ldots, m-1}$ are i.i.d. random variables with common moment generating function $M_{H(\infty, Y)}\left(M_{X}(\cdot)-1\right)$.

Finally, by (31), (32) and (33), we have

$$
\limsup _{m \rightarrow \infty} \frac{1}{m} \log \mathbb{E}\left[e^{\theta A_{m}}\right] \leq \varepsilon \log M_{H(\infty, Y)}\left(M_{X}(\theta)-1\right)+\varepsilon(1-\varepsilon) ;
$$

then, by (S), we conclude letting $\varepsilon$ go to zero.

\section{References}

[AA] Albrecher H. And Asmussen S., Ruin probabilities and aggregate claims distributions for shot noise Cox processes. Scand. Actuar. J., 2006 86-110.

[AN] Asmussen S. And Nielsen H.M., Ruin probabilities via local adjustment coefficients. J. Appl. Probab., 33 (1995) 736-755.

[A] Asmussen S., Ruin Probabilities. World Scientific, London (2000).

[AL] Ausin M.C. And Lopes H.F., Bayesian estimation of ruin probabilities with a heterogeneous and heavy-tailed insurance claim-size distribution. Aust. N. Z. J. Stat. 49 (2007) 415-434.

[AWL] Ausin M.C., Wiper M.P. and Lillo R.E., Bayesian estimation of finite time ruin probabilities. Appl. Stoch. Models Bus. Ind. 25 (2009) 787-805.

[Bo] Borovkov A.A., Boundary values problems for random walks and large deviations for function spaces. Theory Probab. Appl., 12 (1967) 575-595. 
[Br] BrÉmaud P., An insensitivity property of Lundberg's estimate for delayed claims. J. Appl. Prob., 37 (2000) 914-917.

[Bu] Bucklew J.A., Large deviation techniques in decision, simulation, and estimation. Wiley, New York (1990).

[CFT] Cottrell M., Fort J.-C. and Malgouyres G., Large deviations and rare events in the study of stochastic algorithms. IEEE Trans. Automat. Control, 28 (1983) 907-920.

[C] Cox D.R., Some statistical methods connected with series of events. J. Roy. Statist. Soc. Ser. B. 17 (1955) 129-157; discussion, 157-164.

[CI] Cox D.R. and Isham V., Point processes. Chapman \& Hall, London-New York (1980).

[DJ1] Dassios A. AND JANG J-W., Pricing of catastrophe reinsurance and derivatives using the Cox process with shot noise intensity. Finance Stoch., 7 (2003) 73-95.

[DJ2] Dassios A. AND JANG J.-W., Kalman-Bucy filtering for linear systems driven by the Cox process with shot noise intensity and its application to the pricing of reinsurance contracts. J. Appl. Probab., 42 (2005) 93-107.

[d] DE Acosta A., Large deviations for vector valued Lévy processes. Stochastic Process. Appl., 51 (1994) 75-115.

[DZ] Dembo A. And Zeitouni O., Large Deviations Techniques and Applications (2nd Edition). Springer-Verlag, New York (1998).

[D] DJehiche B., A large deviation estimate for ruin probabilities. Scand. Actuar. J., 1993 $42-59$.

[F] Ferguson T.S., A Bayesian analysis of some nonparametric problems. Ann. Statist., 1 (1973), 209-230.

[GGOP] Ganesh A., Green P., O'Connell N. and Pitts S., Bayesian network management. Queueing Systems Theory Appl., 28 (1998) 267-282.

[GMT] Ganesh A., Macci C. And TorRisi G.L., A class of risk processes with reservedependent premium rate: sample path large deviations and importance sampling. Queueing Syst., 55 (2007) 83-94.

[GO1] Ganesh A. and O'Connell N., An inverse of Sanov's theorem. Statist. Probab. Lett. 42 (1999) 201-206.

[GO2] Ganesh A. And O'Connell N., A large deviation principle for Dirichlet posteriors. Bernoulli, 6 (2000) 1021-1034.

[GOW] Ganesh A., O'Connell N. And Wischik D., Big Queues. Lecture Notes in Mathematics 1838, Springer, Berlin (2004).

[G] Gerber, H.U., An Introduction to Mathematical Risk Theory. Huebener Foundation Monograph 8, Univ. Pennsylvania (1979).

[KM1] Klüppelberg C. And Mikosch T., Explosive Poisson shot noise processes with applications to risk reserves. Bernoulli, 1 (1995) 125-147.

[KM2] Klüppelberg C. And Mikosch T., Delay in claim settlement and ruin probability approximations. Scand. Actuar. J., 1995 154-168. 
[K] Kühn C., Shot-Noise Processes. Encyclopedia of Actuarial Sciences. J.L. Teugels and B. Sundt Eds., pp. 1556-1558. Wiley, Chichester (2004).

[LT] Ledoux M. And Talagrand M., Probability in Banach Spaces. Springer, New York (1991).

[LN] Lehtonen T. And Nyrhinen H., Simulating level-crossing probabilities by importance sampling. Adv. in Appl. Probab., 24 (1992) 858-874.

[MP] Macci C. and Petrella L., Mixtures of conjugate prior distributions and large deviations for level crossing probabilities. Sankhyā, 68 (2006) 61-89.

[M] Majewski K., Large deviations for multi-dimensional reflected fractional Brownian motion. Stoch. Stoch. Rep., 75 (2003) 233-257.

[Mo] Møller J., Shot noise Cox processes. Adv. in Appl. Probab., 35 (2003) 614-640.

[MS] Müller A. And Stoyan D., Comparison Methods for Stochastic Models and Risks. John Wiley \& Sons Ltd., Chichester (2002).

[P] Protter P., Stochastic Integration and Differential Equations. Springer, Berlin (1990).

[S] Siegmund D., Importance sampling in the Monte Carlo study of sequential tests. Ann. Statist., 4 (1976) 673-684.

[YGN] Yuen K.C., Guo J. And NG K.W. On ultimate ruin in a delayed-claims risk model. J. Appl. Probab. 42 (2005) 163-174. 